\title{
Phylogeny and taxonomy of the genus Cylindrocladiella
}

\author{
L. Lombard • R. G. Shivas $\cdot$ C. To-Anun $\cdot$ P. W. Crous
}

Received: 10 June 2011 / Revised: 10 November 2011 / Accepted: 25 November 2011

(C) The Author(s) 2012. This article is published with open access at Springerlink.com

\begin{abstract}
The genus Cylindrocladiella was established to accommodate Cylindrocladium-like fungi that have small, cylindrical conidia and aseptate stipe extensions. Contemporary taxonomic studies of these fungi have relied on morphology and to a lesser extent on DNA sequence comparisons of the internal transcribed spacer regions (ITS 1, 2 and 5.8S gene) of the ribosomal RNA and the $\beta$-tubulin gene regions. In the present study, the identity of several Cylindrocladiella isolates collected over two decades was determined using morphology and phylogenetic inference. A phylogeny constructed for these isolates employing the $\beta$-tubulin, histone $\mathrm{H} 3$, ITS, 28S large subunit and translation elongation factor 1-alpha gene regions resulted in the identification of several cryptic species in the genus. In spite of
\end{abstract}

L. Lombard $(\varangle) \cdot$ P. W. Crous

CBS-KNAW Fungal Biodiversity Centre,

Uppsalalaan 8,

3584CT Utrecht, The Netherlands

e-mail: 1.lombard@cbs.knaw.nl

R. G. Shivas

Agri-Science Queensland, Ecosciences Precinct,

Dutton Park,

4102 Queensland, Australia

C. To-Anun

Department of Plant Pathology, Faculty of Agriculture,

Chiang Mai University,

Chiang Mai 50200, Thailand

P. W. Crous

Microbiology, Department of Biology, Utrecht University,

Utrecht, The Netherlands

\section{P. W. Crous}

Wageningen University and Research Centre (WUR),

Laboratory of Phytopathology,

Wageningen, The Netherlands the 18 new Cylindrocladiella species described in this study based on morphological and sequence data, several species complexes remain unresolved.

Keywords Cylindrocladiella . Cryptic species · Phylogeny · Taxonomy

\section{Introduction}

The genus Cylindrocladiella was established by Boesewinkel (1982) to accommodate five Cylindrocladium-like species producing small, cylindrical conidia. Cylindrocladiella, which is based on C. parva, is distinguished from the anamorph state of Calonectria (= Cylindrocladium) by its symmetrically branched conidiophores that can be penicillate and/or subverticillate, producing an asymmetrical bundle of small, cylindrical, 1-septate conidia $(<20 \mu \mathrm{m}$ in length), aseptate stipe extensions, and having Nectricladiella teleomorphs (Boesewinkel 1982, Crous and Wingfield 1993, Schoch et al. 2000). The Nectricladiella teleomorphs are characterised by their perithecia having smooth walls that collapse laterally when dry, and brown setae arising from the perithecial wall surface (Schoch et al. 2000).

Initially, the generic status of Cylindrocladiella was strongly contested (Peerally 1991, Sharma and Mohanan 1991). Morphological evaluations and comparisons by Crous and Wingfield (1993) and Crous et al. (1994), however, confirmed the generic status of this genus, which was later supported by molecular data (Victor et al. 1998, Schoch et al. 2000). Victor et al. (1998) used RFLPs, ATDNA data and morphological comparisons, to recognise seven species in the genus. This was later supported by phylogenetic inference of the ITS and partial $\beta$-tubulin gene regions, resulting in the addition of another species to the 
genus (Schoch et al. 2000). To date, nine species of Cylindrocladiella are recognized, with only two connected to their respective Nectricladiella teleomorph states (Crous 2002, van Coller et al. 2005).

Cylindrocladiella spp. are soil-borne fungi, and are generally regarded as pathogens and/or saprobes of various plant hosts and substrates in temperate, sub-tropical and tropical regions worldwide (Crous 2002, van Coller et al. 2005, Scattolin and Montecchio 2007). They have been associated with a variety of disease symptoms that included leaf spots, and rots of roots, stems and cuttings of agricultural, forestry and horticultural crops (Crous et al. 1991, Peerally 1991, Crous and Wingfield 1993, Victor et al. 1998, Crous 2002, van Coller et al. 2005, Scattolin and Montecchio 2007).

The aim of this study was to consider the identity of several Cylindrocladiella isolates collected over the past two decades from various substrates and regions of the world. To achieve this goal, morphological and culture characteristics were combined with multigene phylogenetic inference for all isolates studied.

\section{Material and methods}

\section{Isolates}

Isolates and ex-type strains of Cylindrocladiella spp. were obtained from various culture collections, isolated from symptomatic plant material and/or baited from soils as described in Crous (2002) and indicated in Table 1. Representative strains have been maintained in the culture collection of the CBSKNAW Fungal Biodiversity Centre (CBS) and the working collection of Pedro Crous (CPC).

\section{Phylogeny}

Total genomic DNA was extracted from 7-10 day old single-conidial cultures using the technique described by Möller et al. (1992). Partial fragments of the following genes and gene regions were amplified using the PCR conditions and primer sets mentioned in Lombard et al. (2010b): $\beta$-tubulin (BTUB), histone H3 (HIS3), internal transcribed spacers and $5.8 \mathrm{~s}$ rDNA (ITS), $28 \mathrm{~s}$ large subunit (LSU) and translation elongation factor 1-alpha (TEF1- $\alpha$ ). The PCR reactions were carried out using a MyCycler ${ }^{\mathrm{TM}}$ thermal cycler (Bio-Rad Laboratories, Inc.) consisting of an initial step of $95^{\circ} \mathrm{C}$ for $5 \mathrm{~min}$ followed by 40 cycles of $30 \mathrm{~s}$ at $95^{\circ} \mathrm{C}, 30 \mathrm{~s}$ at $52^{\circ} \mathrm{C}, 1 \mathrm{~min}$ at $72^{\circ} \mathrm{C}$ and ending with a final extension step of 7 min at $72^{\circ} \mathrm{C}$.

Amplicons were sequenced in both directions using the same primer sets used for amplification and the consensus sequences were aligned using MAFFT v6.611 (Katoh and
Toh 2008) for each gene region. Ambiguous regions in the alignments were removed manually and both ends of the sequences were truncated. All sequences obtained were deposited in GenBank with accession numbers listed in Table 1.

Analyses of the DNA sequence data were done using PAUP (Phylogenetic Analysis Using Parsimony, v4.0b10, Swofford 2002). Initial neighbour-joining analyses (NJ) with the uncorrected ("p"), Juke-Cantor and HKY85 substitution models were done using the LSU sequence data to determine if the Cylindrocladiella isolates used in this study formed a monophyletic group. Congruency of the sequence data for each locus were determined using visual inspection of the tree topologies of $70 \%$ reciprocal NJ bootstrap trees (Gueidan et al. 2007) determined as described in Lombard et al. (2010c). Thereafter, the combined DNA sequence dataset was subjected to maximum parsimony (MP) and Bayesian analyses.

For the MP analysis, the phylogenetic relationships were determined using a heuristic search with 1000 random sequence additions with a tree bisection-reconnection algorithm and the branch swapping option set on "best trees" only. All characters were weighted equally and alignment gaps were treated as missing data. Measures calculated for parsimony included tree length (TL), consistency index (CI), retention index (RI) and rescaled consistence index (RC). Branch support was assessed using a 1000 bootstrap replicates.

For Bayesian analysis, a Markov Chain Monte Carlo (MCMC) algorithm was used to generate phylogenetic trees with posterior probabilities using MrBayes v3.1.1 (Ronquist and Heulsenbeck 2003). Nucleotide substitution models were determined for each gene using the Akaike Information Criterion (AIC) in MrModeltest v2.3 (Nylander 2004) and included in the analysis. The DNA sequence data was subjected to two separate analyses of four MCMC chains run from random trees for 1,000,000 generations with sampling at every 100 generations. Both runs converged on the same likelihood score and tree topology, and therefore the first 1,000 trees were discarded as the burn-in phase. Posterior probabilities were determined from the remaining trees. All alignments and trees generated in this study, have been deposited in TreeBASE (http:/www. treebase.org).

\section{Taxonomy}

Single-conidial cultures of Cylindrocladiella isolates were prepared on synthetic nutrient-poor agar (SNA; Nirenburg 1981) as described in Lombard et al. (2009). In some cases, carnation leaf pieces were added to the SNA to promote sporulation. The gross morphological characteristics were determined with 30 measurements at $\times 1,000$ magnification 


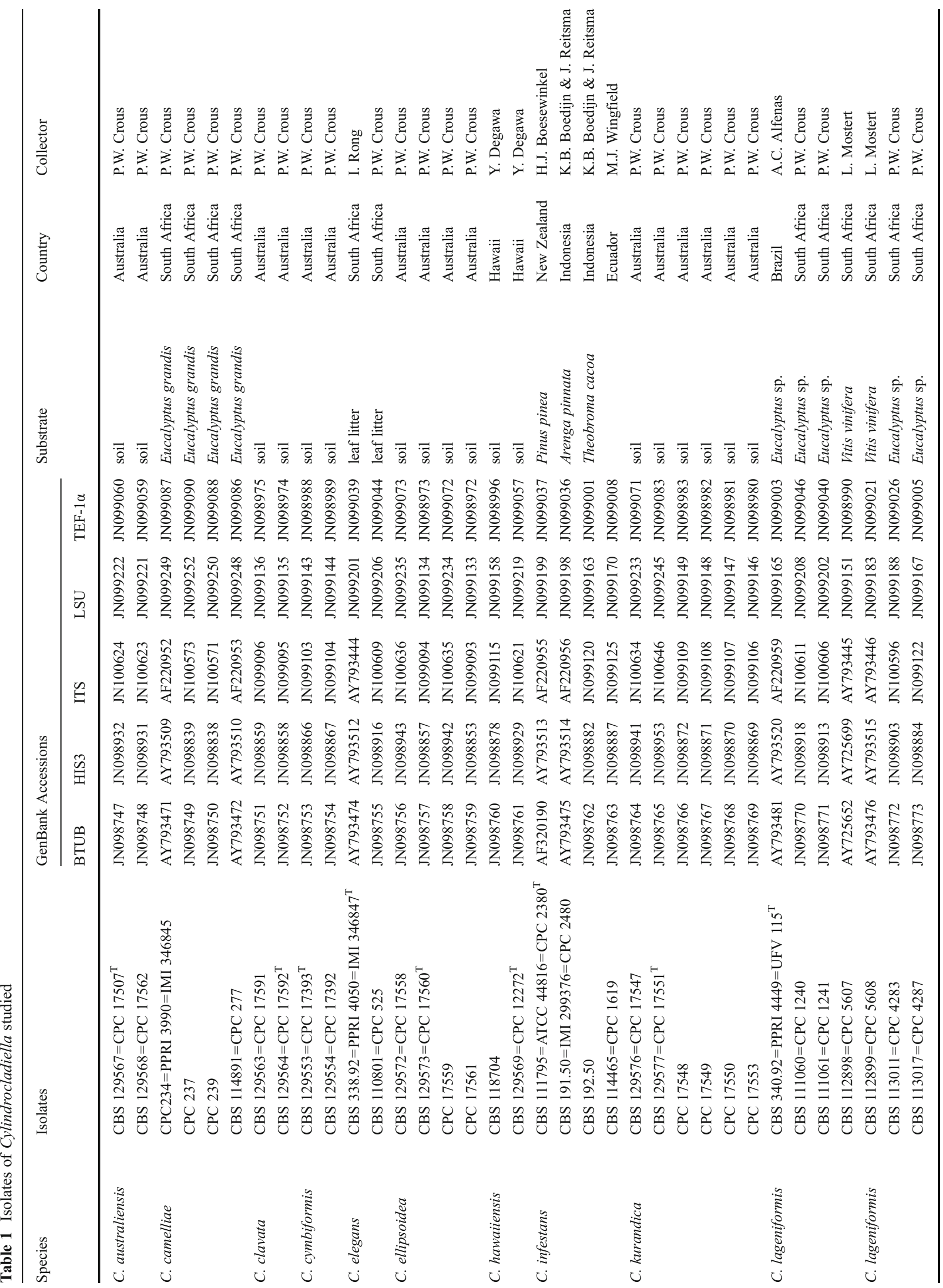




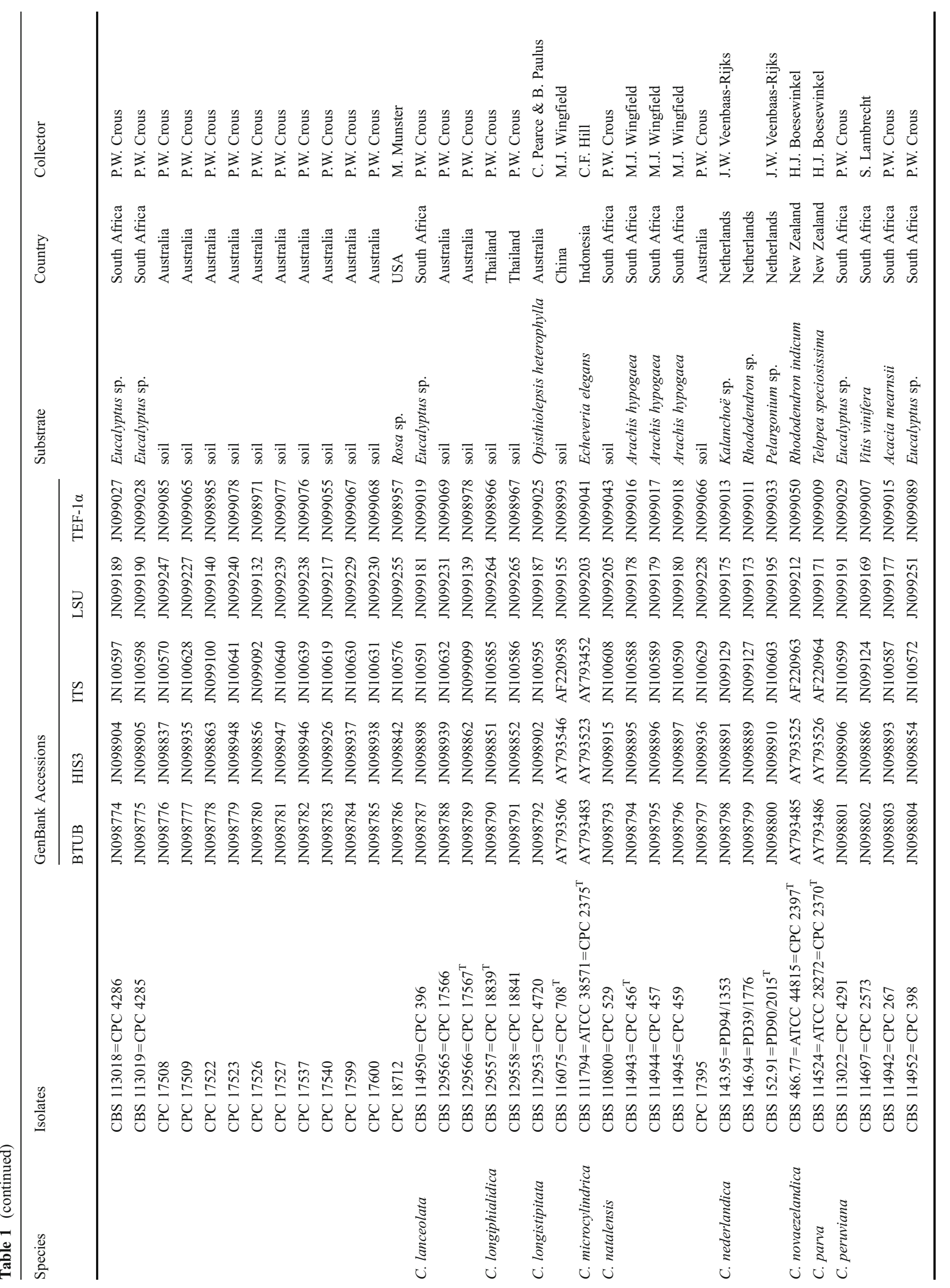




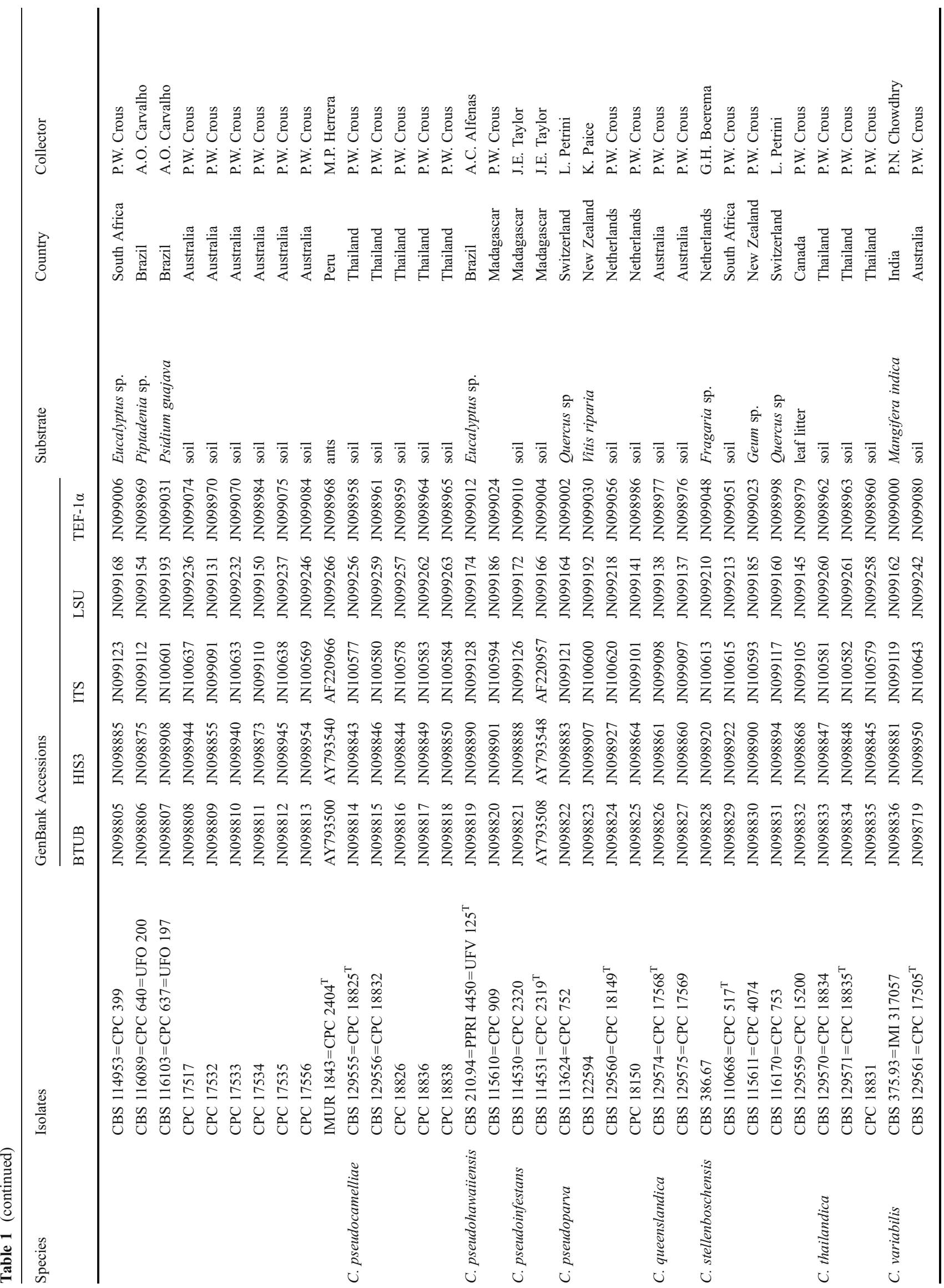




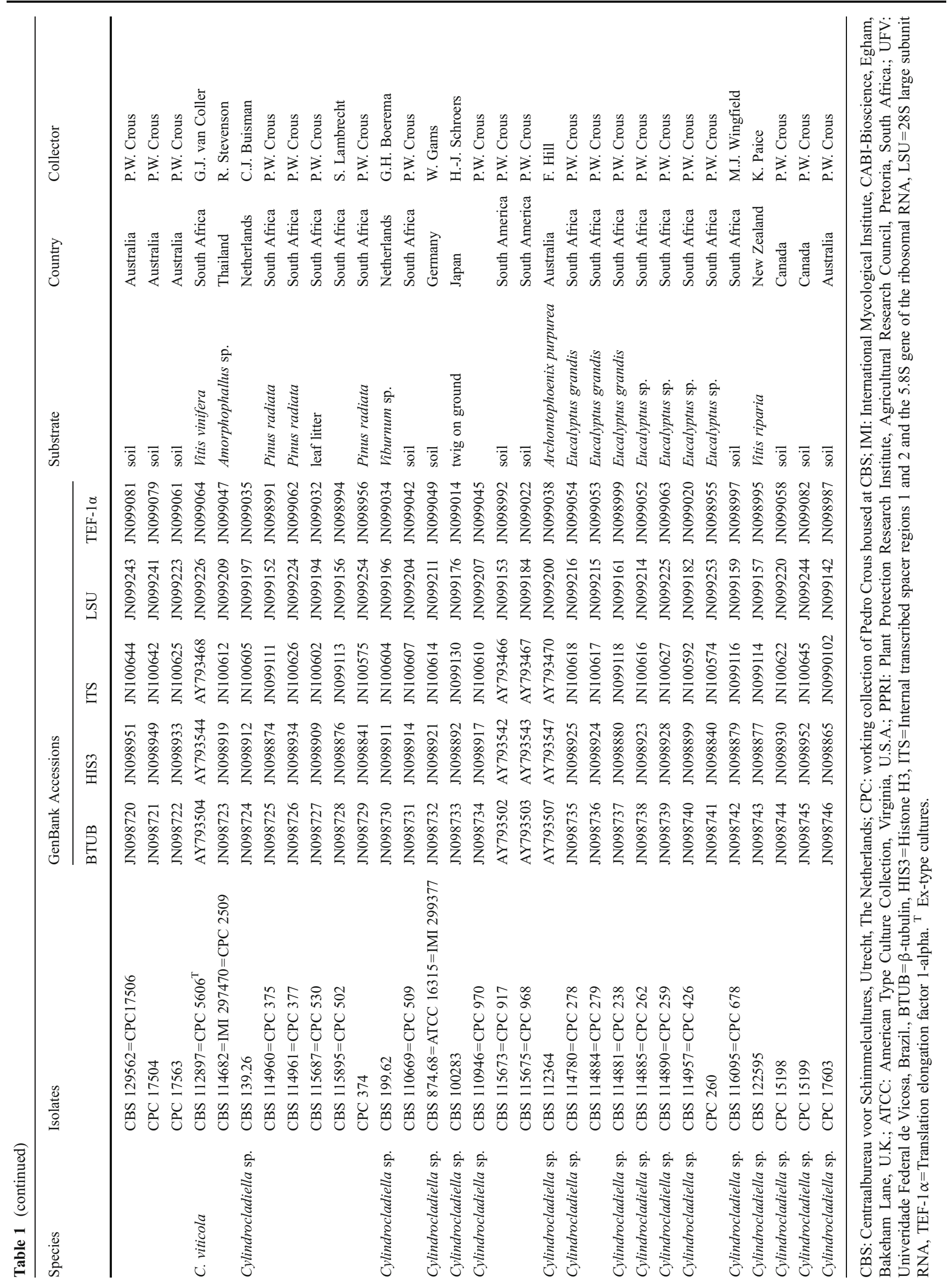


of the fungal structures mounted in $85 \%$ lactic acid. The conidial measurements are presented as the $95 \%$ confidence level with extremes in parentheses. Only the extremes are presented for other structures. The colony colours were determined on $2 \% \mathrm{w} / \mathrm{v}$ malt extract agar (MEA) after 7 day incubation at $24^{\circ} \mathrm{C}$ in the dark using the colour charts of Rayner (1970). Descriptions, nomenclature and illustrations were deposited in MycoBank (Crous et al. 2004).

\section{Results}

Phylogeny

Amplicons of approximately 530 bases were determined for BTUB, HIS3 and TEF- $1 \alpha, 500$ for ITS, and 850 for LSU. The phylogenetic analysis included 136 ingroup taxa, with Ca. pauciramosa (CBS 114861) and Ca. brachiatica (CBS 123700) as outgroup taxa. The initial NJ analysis of the LSU sequence data revealed that all the isolates included in the study formed a monophyletic clade (results not shown). Comparisons of the $70 \%$ reciprocal bootstrap NJ tree topologies of the individual gene regions showed no conflict and therefore the sequence datasets were combined. The resulting dataset of 2,956 characters, including alignment gaps, consisted of 2,070 constant and 131 parsimonyuninformative characters. Analysis of the 755 parsimonyinformative characters yielded 1,224 trees $(\mathrm{TL}=, 4486$; $\mathrm{CI}=$ 0.308 ; $\mathrm{RI}=0.843$; $\mathrm{RC}=0.260$ ), of which the first tree is presented (Fig. 1). For the Bayesian analysis, a HKY $+\mathrm{I}+$ $G$ model was selected for BTUB and TEF- $1 \alpha$, GTR $+\mathrm{I}+\mathrm{G}$ for HIS3 and LSU, and SYM + I + G for ITS which was incorporated into the analysis. The Bayesian consensus tree confirmed both the tree topology and bootstrap support of the strict consensus tree obtained with maximum-parsimony.

In the phylogenetic tree (Fig. 1) the Cylindrocladiella isolates are divided into two main clades. The first main clade [bootstrap support $(\mathrm{BS})=98$; posterior probability $(\mathrm{PP})=0.70]$ is further divided into two subclades. The first subclade $(\mathrm{BS}=98 ; \mathrm{PP}=0.70)$ represents $C$. novaezelandiae (CBS 486.77), C. elegans (CBS 338.92) and other closely related isolates that could represent novel phylogenetic species. The second subclade ( $\mathrm{BS}=100 ; \mathrm{PP}=0.75)$ representing C. camelliae (CPC 234; Crous 2002) and C. peruviana (IMUR 1843) also consists of closely related isolates clustering together in smaller well-supported terminal clades, each representing possible novel species.

The second main clade $(\mathrm{BS}=59 ; \mathrm{PP}=0.53)$ is also divided into two subclades. In the first subclade $(\mathrm{BS}=82 ; \mathrm{PP}=$ 0.90 ) representing $C$. parva (CBS 114524) several isolates form well-supported terminal clades, also indicating potentially new species. The second subclade $(\mathrm{BS}=91 ; \mathrm{PP}=$ $0.93)$ further divides into a clade $(\mathrm{BS}=89 ; \mathrm{PP}=0.65)$ representing $C$. viticola (CBS 112897), and a clade $(\mathrm{BS}=100 ; \mathrm{PP}=1.00)$ containing $C$. lageniformis (CBS 340.92) and $C$. infestans. This clade also consists of several well-supported terminal clades that could represent novel species. The ex-type strain of the anamorph state of $C$. infestans (CBS 111795) clustered (BS $=83$; $\mathrm{PP}=0.97)$ separately from the ex-type strain of the purported teleomorph state of this species (CBS 114531, $\mathrm{BS}=100 ; \mathrm{PP}=0.98$ ), indicating that each ex-type strain represents a distinct species.

\section{Taxonomy}

Based on the phylogenetic inference and morphological observations, numerous Cylindrocladiella isolates included in this study represent novel species. Following the approach of Lombard et al. (2009, 2010a-c) and Crous et al. (2006, 2008, 2009) for other fungal groups, all new species are described in Cylindrocladiella, as this represents the older generic (Boesewinkel 1982), and best established name for this group of fungi.

Cylindrocladiella australiensis L. Lombard \& Crous, sp. nov. - MycoBank MB561676, Fig. 2.

Etymology - Named after the country from where it was collected, Australia.

Cylindrocladiellae infestantis morphologice valde similis, sed conidiis minoribus, $(9-) 11-13(-15) \times 2-4 \mu \mathrm{m}$, distinguitur.

Teleomorph unknown. Conidiophores dimorphic, penicillate and subverticillate, mononematous and hyaline. Penicillate conidiophores (Fig. 2a-e) comprising a stipe, a penicillate arrangement of fertile branches, a stipe extension and a terminal vesicle; stipe septate, hyaline, smooth, 41-96×6-9 $\mu \mathrm{m}$; stipe extension aseptate, straight, 101-152 $\mu \mathrm{m}$ long, thick-walled with one basal septum, terminating in thin-walled, ellipsoidal to fusoid vesicles (Fig. 2j-1), 6-8 $\mu \mathrm{m}$ wide. Penicillate conidiogenous apparatus (Fig. $2 \mathrm{f}-\mathrm{i}$ ) with primary branches aseptate, 13-21× 3-5 $\mu \mathrm{m}$, secondary branches aseptate, $11-15 \times 3-6 \mu \mathrm{m}$, each terminal branch producing 2-4 phialides; phialides cymbiform to cylindrical, hyaline, aseptate, $8-17 \times 2-4 \mu \mathrm{m}$, apex with minute periclinal thickening and collarette. Subverticillate conidiophores (Fig. $2 \mathrm{~m}-\mathrm{n}$ ) abundant, comprising of a septate stipe, primary and secondary branches terminating in 2-3 phialides; primary branches straight, hyaline, $0-1$-septate, $22-54 \times 2-5 \mu \mathrm{m}$, secondary branches straight, hyaline, aseptate, $21-36 \times 4-5 \mu \mathrm{m}$; phialides cymbiform to cylindrical, hyaline, aseptate, $19-40 \times 2-4 \mu \mathrm{m}$, apex with minute periclinal thickening and collarette. Conidia (Fig. 2o) cylindrical, rounded at both ends, straight, 1-septate, (9-)11-13(-15) $\times 2-4 \mu \mathrm{m}$ $($ av. $=12 \times 3 \mu \mathrm{m})$, frequently slightly flattened at the base, held in asymmetrical clusters by colourless slime. 


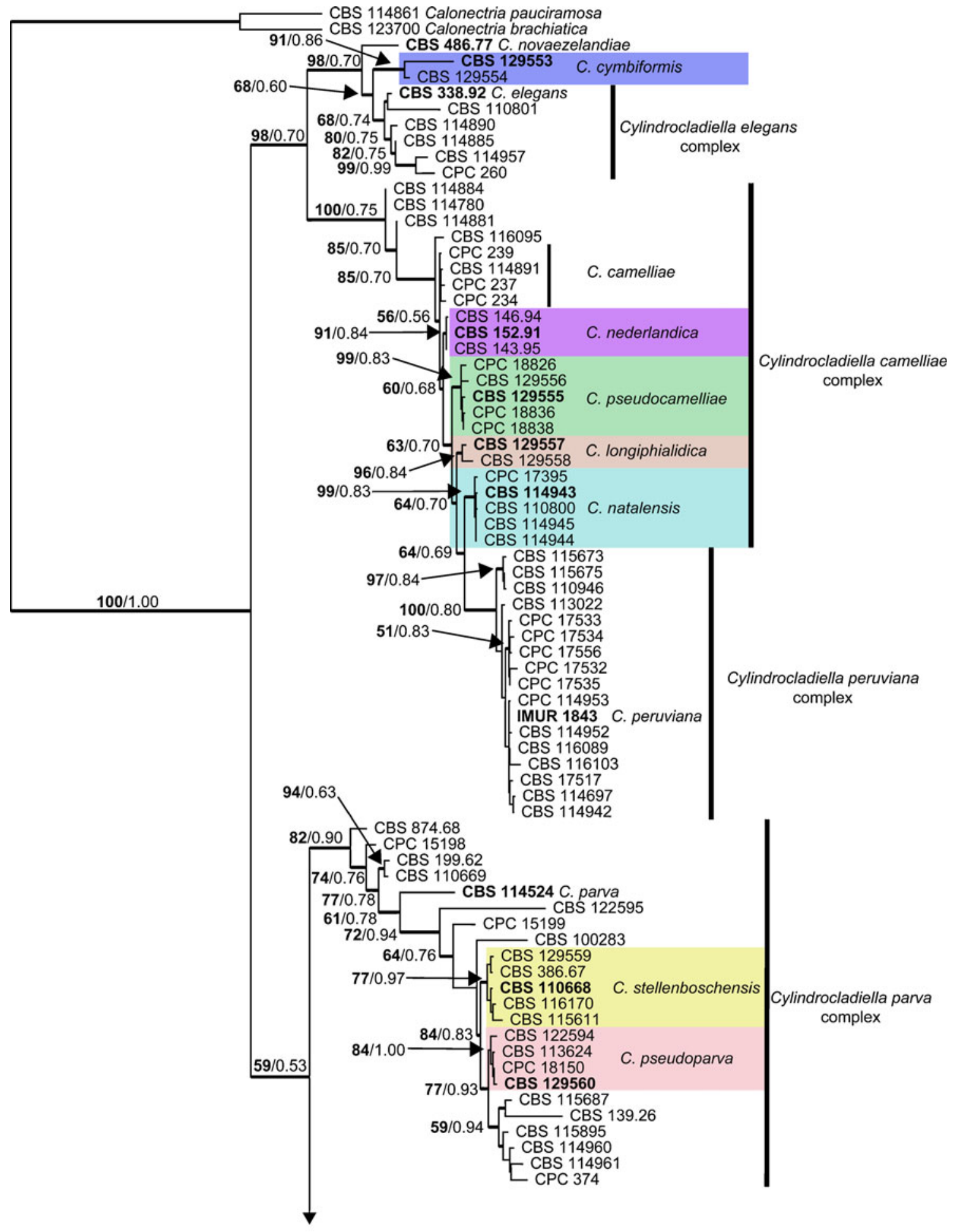

Fig. 1 One of 1,224 most parsimonious trees obtained from a heuristic search with 1,000 random additions sequences of the combined $\beta$-tubulin, histone $\mathrm{H} 3$, internal transcribed spacer regions 1 and 2 and the $5.8 \mathrm{~S}$ gene of the ribosomal RNA, 28S large subunit and translation elongation factor-1alpha sequence alignments of the Cylindrocladiella isolates. Scale bar shows 10 changes and bootstrap support values (bold) from 1,000 replicates and Bayesian posterior probability values are indicated at the nodes. Thickened lines indicate branches in the strict consensus tree and the consensus tree of the Bayesian analyses. Ex-type strains are indicated in bold and coloured block indicate the novel species described. The tree was rooted to Calonectria brachiatica (CBS123700) and Ca. pauciramosa (CBS 114861). Species complexes are indicated on the right 


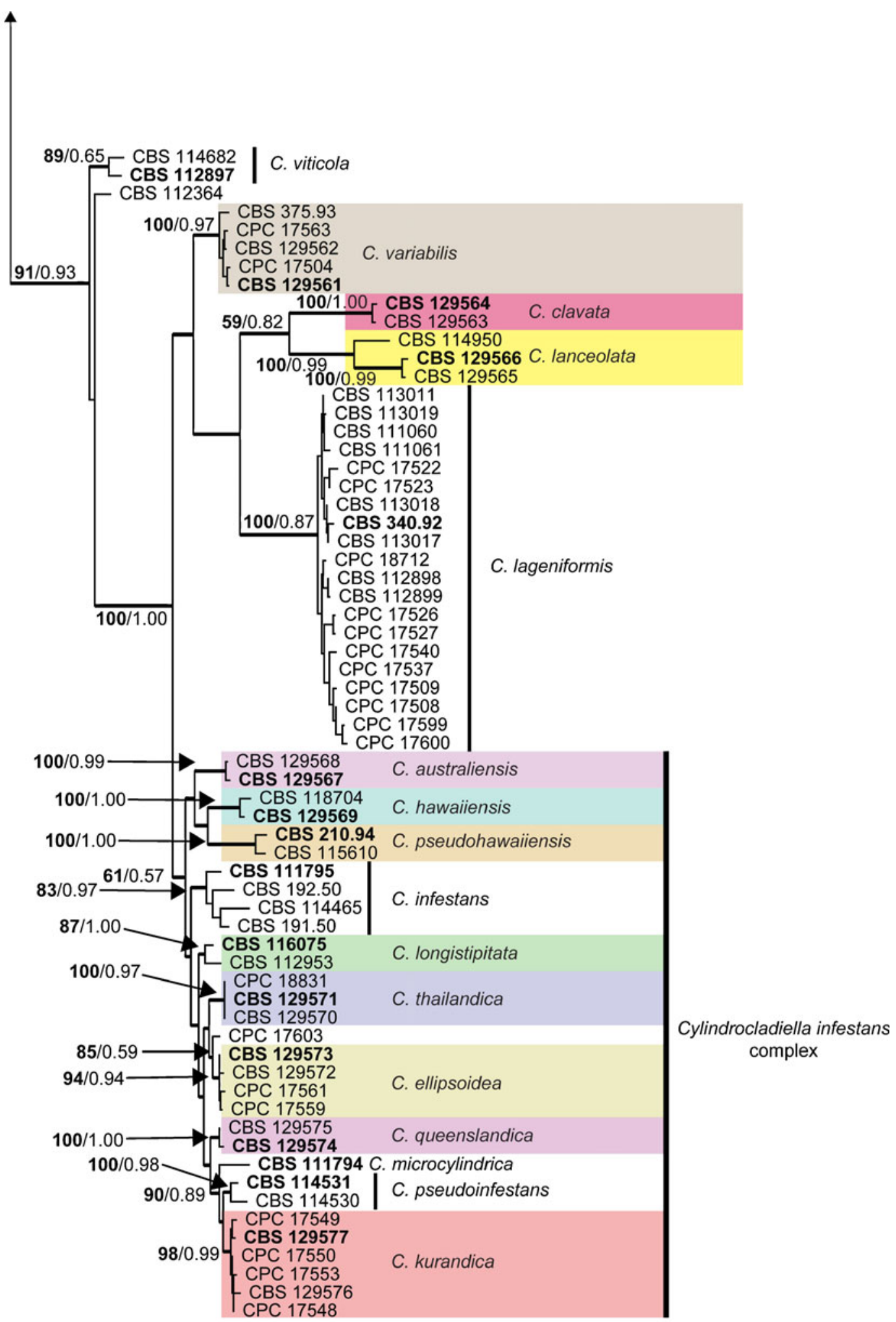

$-^{10}$

Fig. 1 (continued) 

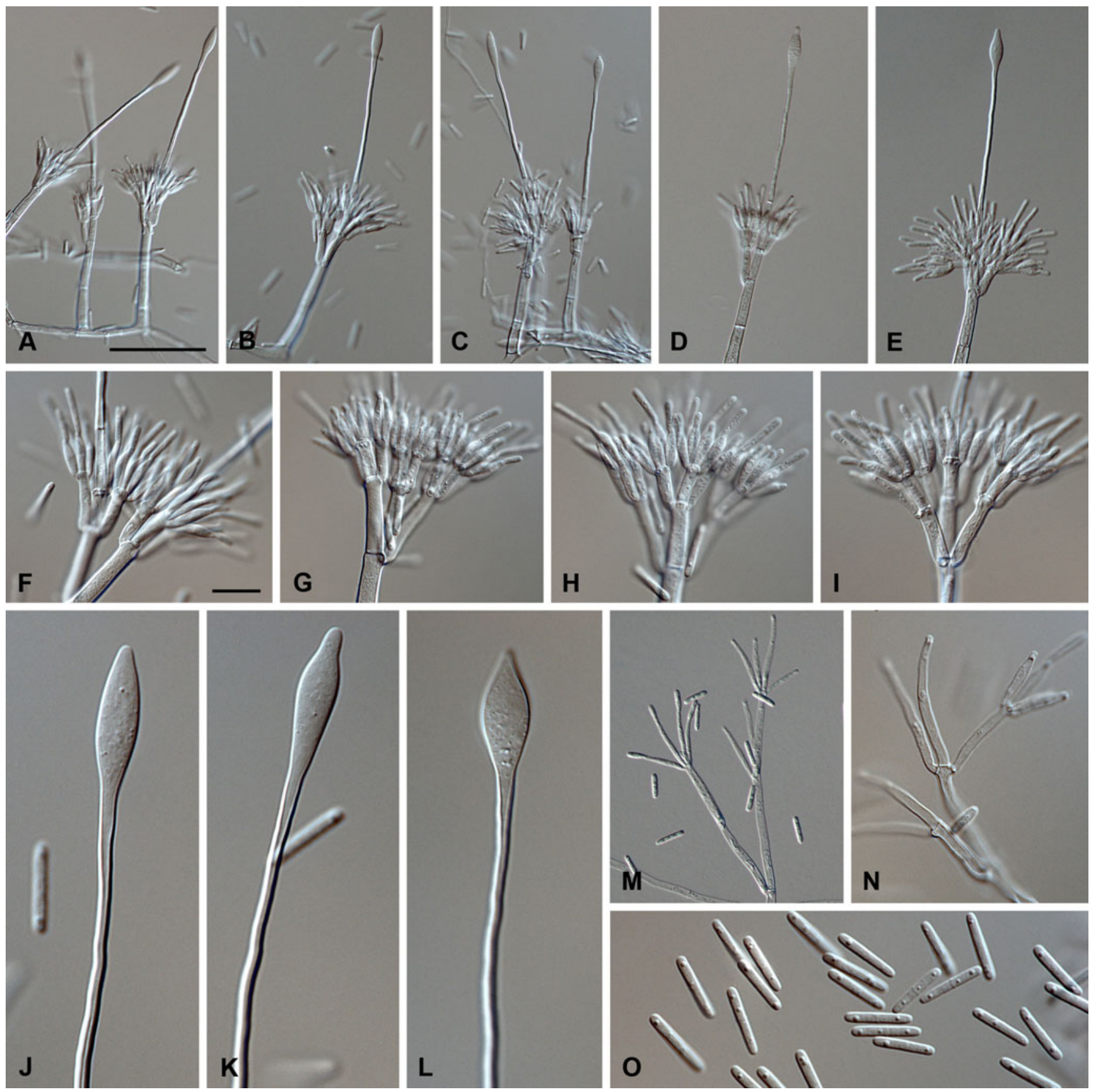

Fig. 2 Cylindrocladiella australiensis. a-e. Penicillate conidiophores. $\mathbf{f}-\mathbf{i}$. Conidiogenous apparatus with conidiophore branches and phialides. $\mathbf{j}-\mathbf{l}$. Terminal vesicles. $\mathbf{m}-\mathbf{n}$. Subverticillate conidiophores. $\mathbf{0}$. Conidia. A=50 $\mu \mathrm{m}$ (apply to $\mathbf{b}-\mathbf{e}, \mathbf{m}$ ), $\mathrm{F}=10 \mu \mathrm{m}$ (apply to $\mathbf{g}-\mathbf{l}, \mathbf{n}-\mathbf{0}$ )

Culture characteristics - Colonies raised (convex), cottony, with smooth margins, white, buff yellow (19 d) to umber (13i) (reverse); chlamydospores moderate throughout medium, arranged in chains; reaching $90 \mathrm{~mm}$ after 7 days on MEA at $24^{\circ} \mathrm{C}$ in the dark.

Specimens examined - Australia, Queensland, Daydream Island, Whitsundays Island Resort, from soil, 2 Aug. 2009, P.W. Crous, holotype CBS-H20596, culture ex-type CBS $129567=$ CPC 17507; Australia, Queensland, Lake Barrine, from soil, 18 June 2009, P.W. Crous, culture CBS $129568=$ CPC 17562.

Notes - Cylindrocladiella australiensis can be distinguished from $C$. infestans (av. $15 \times 3 \mu \mathrm{m}$ ) by its smaller conidia and its terminal vesicle shape. The subverticillate conidiophores of $C$. australiensis also form secondary branches not reported for $C$. infestans. Unique fixed nucleotides were also identified for $C$. australiensis for three loci: BTUB positions 186 (T), 296 (C), 350 (T), 
381 (C) and 387 (T); HIS3 positions 90 (T) and 387 (T); TEF$1 \alpha$ positions $113(\mathrm{C}), 153(\mathrm{G}), 155(\mathrm{C}), 168(\mathrm{~T}), 229(\mathrm{~T}), 232$ (C), $254(\mathrm{G}), 266(\mathrm{C}), 282(\mathrm{~T}), 462(\mathrm{~A})$ and $468(\mathrm{C})$.

Cylindrocladiella clavata L. Lombard \& Crous, sp. nov. MycoBank MB561674, Fig. 3.

Etymology - Named after the clavate shape of its vesicles.

Cylindrocladiellae variabilis morphologice similis, sed vesiculis clavatis distinguitur.

Teleomorph unknown. Conidiophores monomorphic, penicillate, mononematous and hyaline. Penicillate conidiophores (Fig. 3a-e) comprising a stipe, a penicillate arrangement of fertile branches, a stipe extension and a terminal vesicle; stipe septate, hyaline, smooth, 40-86×6$10 \mu \mathrm{m}$; stipe extension aseptate, straight, 116-170 $\mu \mathrm{m}$ long, thick-walled with one basal septum, terminating in thin-walled, elongated, clavate vesicles (Fig. 3f-h), 4-7 $\mu \mathrm{m}$ wide. Penicillate conidiogenous apparatus (Fig. 3i-k) with primary branches aseptate, $10-23 \times 3-8 \mu \mathrm{m}$, secondary branches aseptate, $6-11 \times 2-4 \mu \mathrm{m}$, each terminal branch producing 2-4 phialides; phialides doliiform to cymbiform, hyaline, aseptate, $7-12 \times 2-3 \mu \mathrm{m}$, apex with minute periclinal thickening and collarette absent. Subverticillate conidiophores not observed. Conidia (Fig. 31) cylindrical, rounded at both ends, straight, 1-septate, (10-)13-15($16) \times 2-3 \mu \mathrm{m}($ av. $=14 \times 2 \mu \mathrm{m})$, frequently slightly flattened at the base, held in asymmetrical clusters by colourless slime.

Culture characteristics - Colonies raised (convex), cottony, with undulate margins, white with buff yellow (19 d) centre, umber (13i) (reverse); chlamydospores extensive throughout medium arranged in chains; reaching $70 \mathrm{~mm}$ after 7 days on MEA at $24^{\circ} \mathrm{C}$ in the dark.

Specimen examined - Australia, Queensland, Byron Bay, from soil, 17 July 2009, P.W. Crous, holotype CBS-H20597, culture ex-type CBS 129564=CPC 17592, Australia, Queensland, Byron Bay, from soil, 17 July 2009, P.W. Crous, culture CBS 129563=CPC 17591.
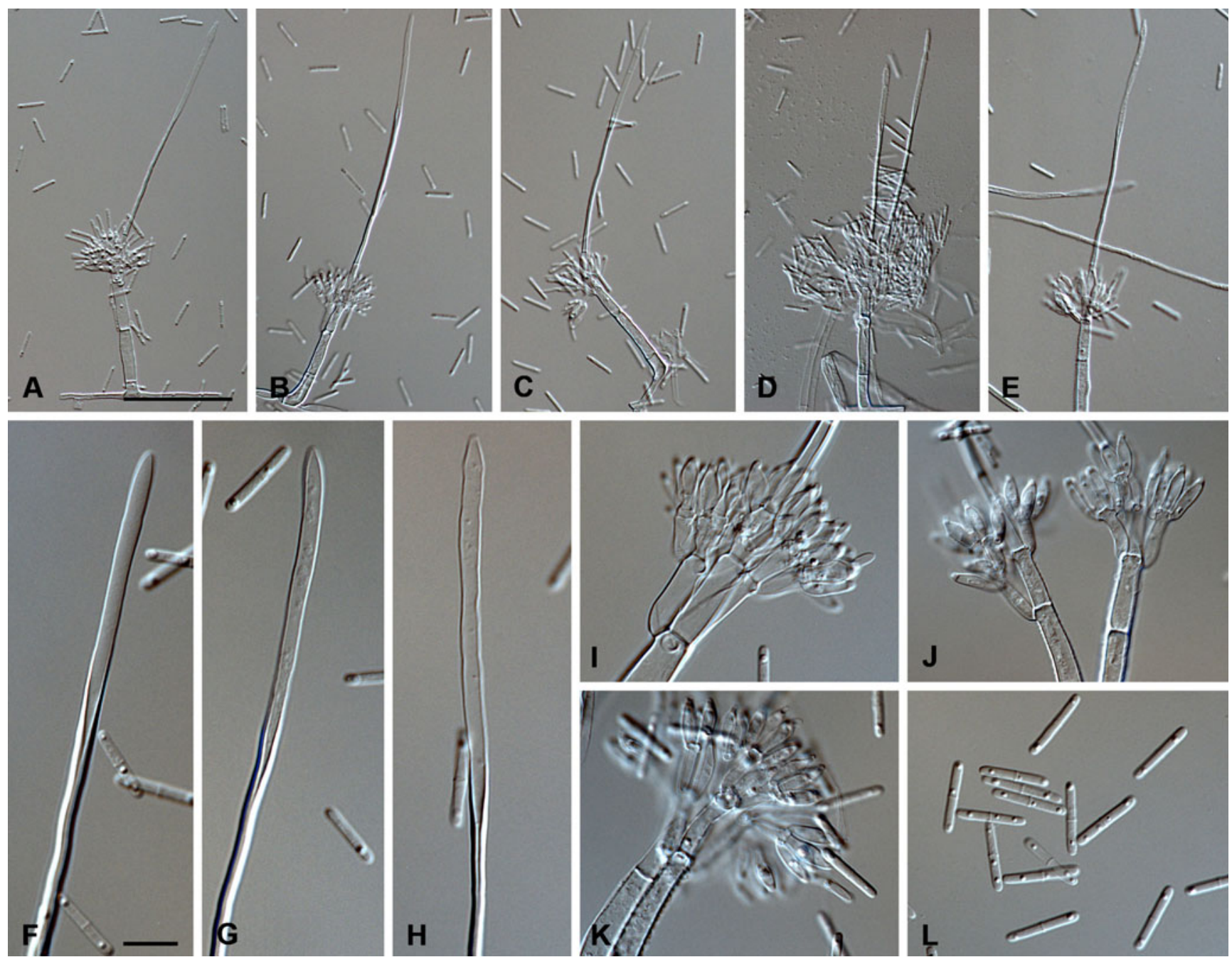

Fig. 3 Cylindrocladiella clavata. a-e. Penicillate conidiophores. $\mathbf{f}-\mathbf{h}$. Terminal vesicles. $\mathbf{i}-\mathbf{k}$. Conidiogenous apparatus with conidiophore branches and phialides. L. Conidia. Scale bars: A=50 $\mu \mathrm{m}$ (apply to $\mathbf{b}-\mathbf{e}$ ), $\mathrm{F}=10 \mu \mathrm{m}$ (apply to $\mathbf{g}-\mathbf{I}$ ) 
Notes - This species can be distinguished from other species in the genus by its elongated clavate terminal vesicles. The conidia are also slightly larger than those of C. lageniformis (av. $12 \times 2 \mu \mathrm{m}$ ).

Cylindrocladiella cymbiformis L. Lombard \& Crous, sp. nov. - MycoBank MB561666, Fig. 4.

Etymology - Named after its phialides, which are cymbiform in shape.

Cylindrocladiellae elegantis morphologice valde similis, sed conidis majoribus, (15-)16-20(-22) $\times(2-) 3-5(-6) \mu \mathrm{m}$, distinguitur.

Teleomorph unknown. Conidiophores monomorphic, penicillate, mononematous, hyaline; comprising a stipe, a penicillate arrangement of fertile branches, a stipe extension and a terminal vesicle (Fig. 4a-e); stipe septate, hyaline, smooth, 44-84 $\times 2-4 \mu \mathrm{m}$; stipe extension aseptate, straight, 107-175 $\mu \mathrm{m}$ long, thick-walled with one basal septum, terminating in thin-walled, lageniform to broadly clavate vesicles (Fig. 4j-1), 6-8 $\mu$ m wide. Penicillate conidiogenous apparatus (Fig. 4f- $\mathrm{i}$ ) with primary branches aseptate, 12 $28 \times 4-6 \mu \mathrm{m}$, secondary branches aseptate, $11-19 \times 2-5 \mu \mathrm{m}$, each terminal branch producing 2-4 phialides; phialides cymbiform, hyaline, aseptate, $11-19 \times 2-5 \mu \mathrm{m}$, apex with minute periclinal thickening and collarette. Subverticillate conidiophores not observed. Conidia (Fig. 4m) cylindrical, rounded at both ends, straight, 1-septate, (15-)16-20($22) \times(2-) 3-5(-6) \mu \mathrm{m}($ av. $=18 \times 3 \mu \mathrm{m})$, frequently slightly flattened at the base, held in asymmetrical clusters by colourless slime.

Culture characteristics - Colonies raised (convex), cottony, with undulate margins, white, buff yellow (19 d) (reverse); chlamydospores sparse throughout medium, arranged in chains; reaching $45 \mathrm{~mm}$ after 7 days on MEA at $24^{\circ} \mathrm{C}$ in the dark.

Specimen examined - Australia, Queensland, Brisbane, from soil, 11 July 2009, P.W. Crous, holotype CBS-H20598,
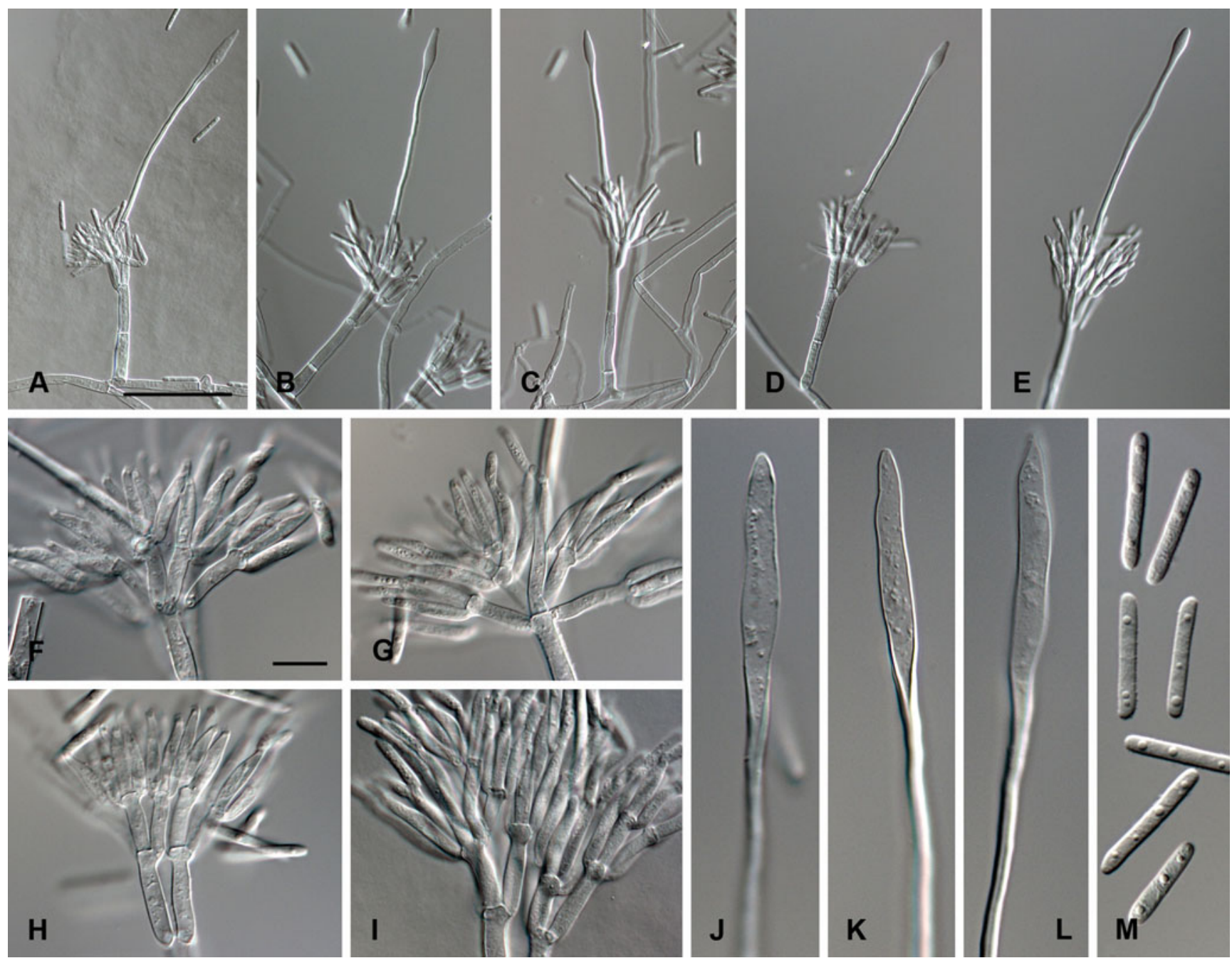

Fig. 4 Cylindrocladiella cymbiformis. a-e. Penicillate conidiophores. $\mathbf{f}-\mathbf{i}$. Conidiogenous apparatus with conidiophore branches and phialides. $\mathbf{j}-\mathbf{l}$. Terminal vesicles. $\mathbf{m}$. Conidia. Scale bars: $\mathrm{A}=50 \mu \mathrm{m}$ (apply to $\mathbf{b}-\mathbf{e}$ ), $\mathrm{F}=10 \mu \mathrm{m}$ (apply to $\mathbf{g}-\mathbf{m}$ ) 
culture ex-type CBS 129553=CPC 17393; Australia, Queensland, Brisbane, from soil, 11 July 2009, P.W. Crous, culture CBS 129554=CPC 17392.

Notes - Based on phylogenetic inference, C. cymbiformis is placed in the $C$. elegans species complex, and closely related to $C$. novaezelandiae. Morphologically, this species has larger conidia (av. $18 \times 3 \mu \mathrm{m}$ ) than $C$. elegans (av. $14.5 \times$ $2 \mu \mathrm{m}$ ) and C. novaezelandiae (av. $14.5 \times 2 \mu \mathrm{m}$ ) (Crous 2002), and stipe extensions are also much longer. Only cymbiform phialides were observed for C. cymbiformis, whereas both $C$. elegans and $C$. novaezelandiae also produce doliiform to reniform phialides. Furthermore, no subverticillate conidiophores were observed for $C$. cymbiformis, but have been reported for C. elegans.

Cylindrocladiella ellipsoidea L. Lombard \& Crous, sp. nov. - MycoBank MB561681, Fig. 5.

Etymology - Named after the characteristic ellipsoid shape of its vesicles.

Cylindrocladiellae infestantis morphologice similis, sed vesiculis clavatis vel ellipsoideis distiunguitur.

Teleomorph unknown. Conidiophores dimorphic, penicillate and subverticillate, mononematous and hyaline. Penicillate conidiophores (Fig. 5a-e) comprising a stipe, a penicillate arrangement of fertile branches, a stipe extension and a terminal vesicle; stipe septate, hyaline, smooth, 41$83 \times 6-8 \mu \mathrm{m}$; stipe extension aseptate, straight, $77-155 \mu \mathrm{m}$ long, thick-walled with one basal septum, terminating in thin-walled, clavate to ellipsoidal vesicles (Fig. 5j-n), 5$8 \mu \mathrm{m}$ wide. Penicillate conidiogenous apparatus (Fig. $5 \mathrm{f}-\mathrm{i}$ ) with primary branches aseptate, 11-20×3-6 $\mu \mathrm{m}$, secondary branches aseptate, $9-12 \times 3-5 \mu \mathrm{m}$, each terminal branch producing 2-4 phialides; phialides doliiform to cymbiform, hyaline, aseptate, $9-12 \times 2-4 \mu \mathrm{m}$, apex with minute periclinal thickening and collarette. Subverticillate conidiophores (Fig. 5o-p) in moderate numbers, comprising of a septate stipe, primary and secondary branches terminating in 2-3 phialides; primary branches straight, hyaline, 0 1-septate, 23-40 $\times 3-6 \mu \mathrm{m}$, secondary branches rare, straight, hyaline, aseptate, $16-31 \times 4 \mu \mathrm{m}$; phialides cymbiform to cylindrical, hyaline, aseptate, $27-52 \times 2-4 \mu \mathrm{m}$, apex with minute periclinal thickening and collarette. Conidia (Fig. 5q) cylindrical, rounded at both ends, straight, 1-septate, (14-)16$18(-19) \times 3-4 \mu \mathrm{m}(\mathrm{av} .=17 \times 4 \mu \mathrm{m})$, frequently slightly flattened at the base, held in asymmetrical clusters by colourless slime.

Culture characteristics - Colonies raised (convex), cottony, with smooth to undulate margins, white, buff yellow (19d) to umber (13i) (reverse); chlamydospores moderate throughout media arranged in chains; reaching $60 \mathrm{~mm}$ after 7 days on MEA at $24^{\circ} \mathrm{C}$ in the dark.

Specimen examined - Australia, Queensland, Lake Barrine, from soil, 18 June 2009, P.W. Crous, holotype CBS-H20599, culture ex-type CBS 129573=CPC 17560;
Australia, Queensland, Lake Barrine, from soil, 18 June 2009, P.W. Crous, culture CBS $129572=$ CPC 17558; Australia, Queensland, Lake Barrine, from soil, 18 June 2009, P.W. Crous, culture CPC 17559.

Notes - Cylindrocladiella ellipsoidea produces subverticillate conidiophores with secondary branches, which has not reported been observed for other species in the $C$. infestans complex. Furthermore, C. ellipsoidea can also be distinguished for others in the complex based on their terminal vesicle shape. Unique fixed nucleotides were also identified for $C$. ellipsoidea for two loci: HIS3 positions 124 (C), 130 (A), 134 (C), 314 (A) and 349 (A); TEF-1 $\alpha$ position 210 (indel).

Cylindrocladiella hawaiiensis L. Lombard \& Crous, sp. nov. - MycoBank MB561677, Fig. 6.

Etymology - Named after Hawaii, where this fungus was collected.

Cylindrocladiellae infestantis morphologice similis, sed vesiculis clavatis distinguitur.

Teleomorph unknown. Conidiophores dimorphic, penicillate and subverticillate, mononematous and hyaline. Penicillate conidiophores (Fig. 6a-c) comprising a stipe, a penicillate arrangement of fertile branches, a stipe extension and a terminal vesicle; stipe septate, hyaline, smooth, 47$80 \times 5-6 \mu \mathrm{m}$; stipe extension aseptate, straight, $80-116 \mu \mathrm{m}$ long, thick-walled with one basal septum, terminating in thin-walled, clavate vesicles (Fig. 6d-e), 5-7 $\mu \mathrm{m}$ wide. Penicillate conidiogenous apparatus (Fig. $6 \mathrm{f}-\mathrm{j}$ ) with primary branches aseptate, $11-19 \times 4-5 \mu \mathrm{m}$, secondary branches aseptate, $8-19 \times 3-4 \mu \mathrm{m}$, each terminal branch producing 2 4 phialides; phialides cymbiform to cylindrical, hyaline, aseptate, $8-18 \times 2-3 \mu \mathrm{m}$, apex with minute periclinal thickening and collarette. Subverticillate conidiophores (Fig. 6k-1) abundant, comprising of a septate stipe, and primary branches terminating in 2-3 phialides; primary branches straight, hyaline, $0-1$-septate, $23-38 \times 3-5 \mu \mathrm{m}$; phialides cymbiform to cylindrical, hyaline, aseptate, $19-41 \times 2-4 \mu \mathrm{m}$, apex with minute periclinal thickening and collarette. Conidia (Fig. $6 \mathrm{~m}$ ) cylindrical, rounded at both ends, straight, 1septate, $(10-) 12-14 \times 2-4 \mu \mathrm{m}(\mathrm{av} .=13 \times 3 \mu \mathrm{m})$, frequently slightly flattened at the base, held in asymmetrical clusters by colourless slime.

Culture characteristics - Colonies raised (convex), cottony, with smooth margins, white, buff yellow (19 d) (reverse); chlamydospores sparse throughout medium, arranged in chains; reaching $65 \mathrm{~mm}$ after 7 days on MEA at $24^{\circ} \mathrm{C}$ in the dark.

Specimens examined - Hawaii, from soil, 1 Aug. 2005, Y. Degawa, holotype CBS-H20600, culture ex-type CBS 129569=CPC 12272; Hawaii, Kaua'i Island, Secret waterfall, from soil, 8 Aug. 2005, Y. Degawa, culture CBS 118704.

Notes - Cylindrocladiella hawaiiensis produces clavate terminal vesicles, distinguishing it from $C$. infestans, which 

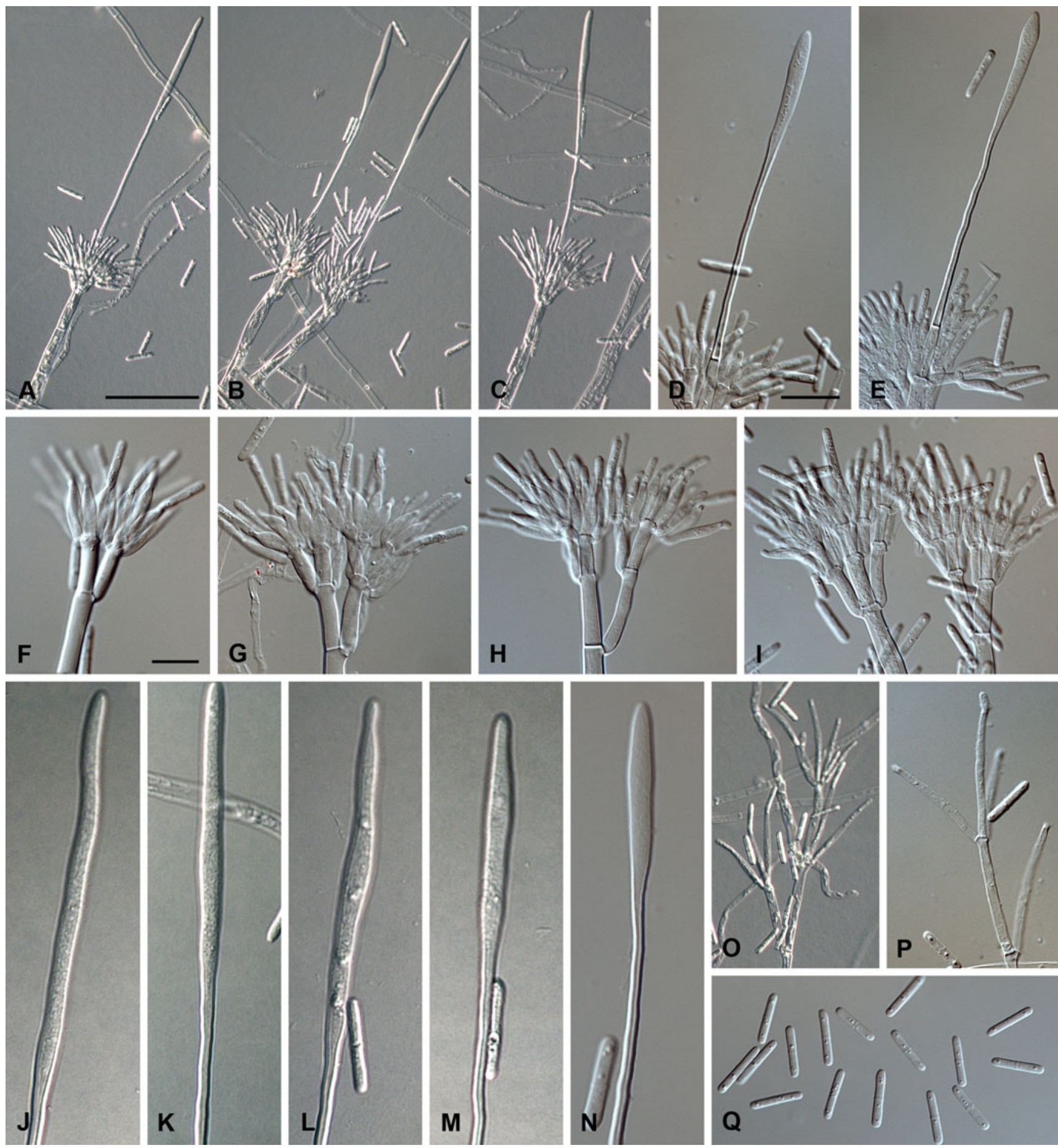

Fig. 5 Cylindrocladiella ellipsoidea. a-e. Penicillate conidiophores. f-i. Conidiogenous apparatus with conidiophore branches and phialides. $\mathbf{j}-\mathbf{n}$. Terminal vesicles. $\mathbf{o}-\mathbf{p}$. Subverticillate conidiophores. q.

has cylindrical terminal vesicles. Conidia of $C$. hawaiiensis (av. $=13 \times 3 \mu \mathrm{m}$ ) are also smaller than those of $C$. infestans (av. $15 \times 3 \mu \mathrm{m}$ ). Unique fixed nucleotides were also identified for C. hawaiiensis for two loci: BTUB positions 91 (A), $113(\mathrm{C}), 121(\mathrm{~T}), 138(\mathrm{~T}), 367(\mathrm{~T}), 368(\mathrm{~T}), 369(\mathrm{C})$ and 375 (G); HIS3 positions 43 (T), 50 (T), 88 (A), 91 (indel), 116
Conidia. Scale bars: $A=50 \mu \mathrm{m}$ (apply to $\mathbf{b}-\mathbf{c}$ ), $\mathrm{D}=20 \mu \mathrm{m}$ (apply to $\mathbf{e}$, $\mathbf{o}), \mathrm{F}=10 \mu \mathrm{m}$ (apply to $\mathbf{g}-\mathbf{n}, \mathbf{p}-\mathbf{q})$

(A), 229 (A), 235 (G), 248 (A), 278 (A), 456 (indel), 462 (T) and 469 (A).

Cylindrocladiella kurandica L. Lombard \& Crous, sp. nov. - MycoBank MB561683, Fig. 7.

Etymology - Named after the Kuranda, the town where this fungus was collected. 

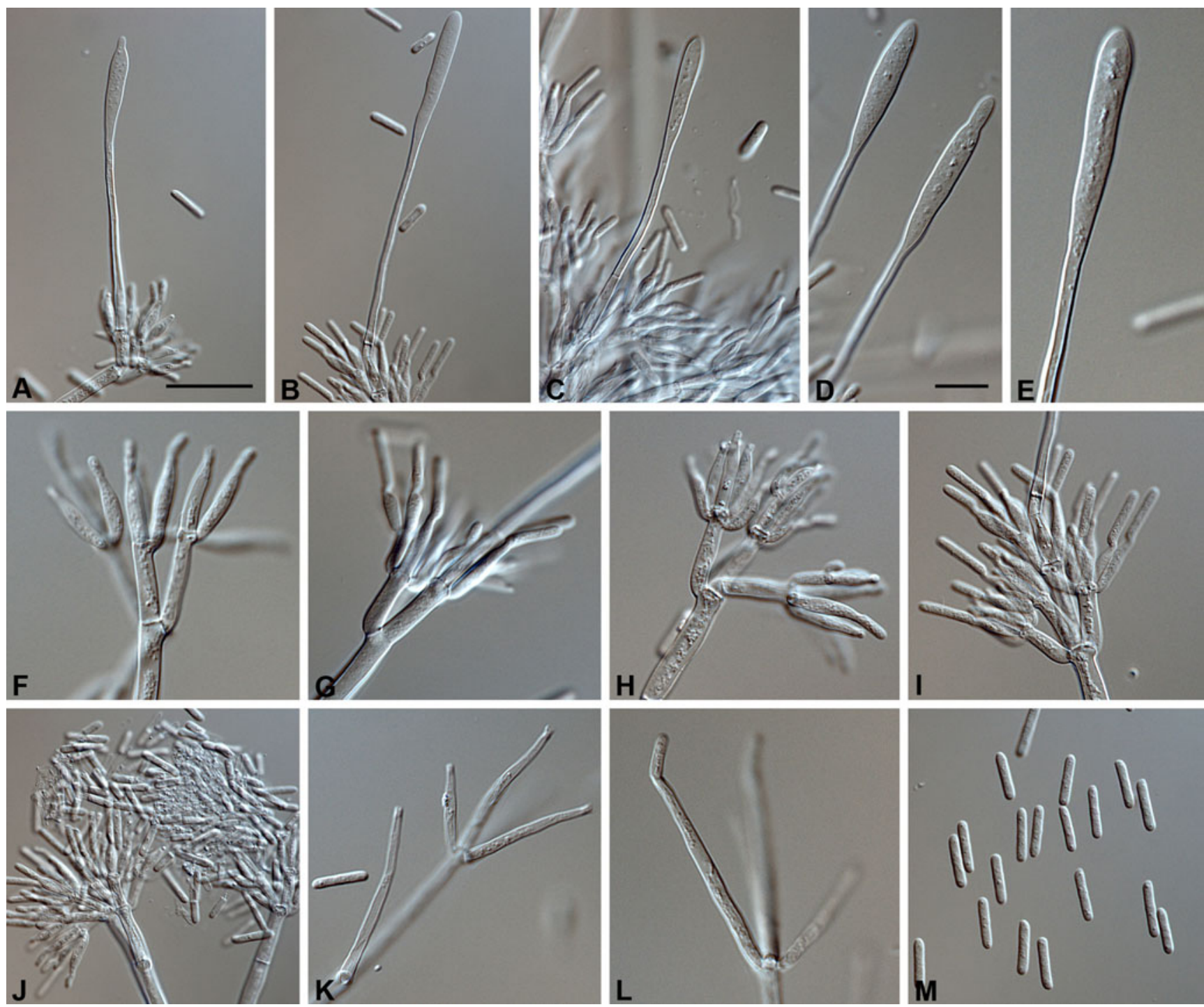

Fig. 6 Cylindrocladiella hawaiiensis. a-c. Penicillate conidiophores. $\mathbf{d}-\mathbf{e}$. Terminal vesicles. $\mathbf{f}-\mathbf{j}$. Conidiogenous apparatus with conidiophore branches and phialides. $\mathbf{k}-\mathbf{l}$. Subverticillate conidiophores. $\mathbf{m}$. Conidia. A=20 $\mathrm{m}$ (apply to $\mathbf{b}, \mathbf{j}$ ), $\mathrm{D}=10 \mu \mathrm{m}$ (apply to $\mathbf{e}-\mathbf{i}, \mathbf{k}-\mathbf{m})$

Cylindrocladiellae infestantis morphologice valde similis et vix distinguibilis, sed characteribus sequentibus nucleotiditis fixationibus in positionibus diversis [BTUB 97 (T), 395 (A) et 482 (T); HIS3 22 (T), 50 (A) et 315 (T); TEF$1 \propto 107(\mathrm{C})]$ genetice distinguitur.

Teleomorph unknown. Conidiophores dimorphic, penicillate and subverticillate, mononematous and hyaline. Penicillate conidiophores (Fig. 7a-e) comprising a stipe, a penicillate arrangement of fertile branches, a stipe extension and a terminal vesicle; stipe septate, hyaline, smooth, 54-87 $\times 5-9 \mu \mathrm{m}$; stipe extension aseptate, straight, 153-219 $\mu \mathrm{m}$ long, thick-walled with one basal septum, terminating in thin-walled, ellipsoidal to lanceolate vesicles (Fig. 7j-1), 6-9 $\mu \mathrm{m}$ wide. Penicillate conidiogenous apparatus (Fig. $7 \mathrm{f}-\mathrm{i}$ ) with primary branches aseptate, 12-24× 3-7 $\mu \mathrm{m}$, secondary branches aseptate, $8-15 \times 2-4 \mu \mathrm{m}$, each terminal branch producing 2-4 phialides; phialides doliiform to cymbiform, hyaline, aseptate, $8-14 \times 2-4 \mu \mathrm{m}$, apex with minute periclinal thickening and collarette. Subverticillate conidiophores (Fig. $7 \mathrm{~m}-\mathrm{O}$ ) in moderate numbers, comprising of a septate stipe, and primary branches terminating in 2-3 phialides; primary branches straight, hyaline, $0-1$-septate, 20 $48 \times 2-4 \mu \mathrm{m}$; phialides cymbiform to cylindrical, hyaline, aseptate, $18-35 \times 2-5 \mu \mathrm{m}$, apex with minute periclinal thickening and collarette. Conidia (Fig. 7p) cylindrical, rounded at both ends, straight, 1-septate, (10-)12-14($16) \times 2-4 \mu \mathrm{m}(\mathrm{av} .=13 \times 3 \mu \mathrm{m})$, frequently slightly flattened at the base, held in asymmetrical clusters by colourless slime.

Culture characteristics - Colonies raised, cottony, with undulate margins, white with straw $(21 \mathrm{~d})$ tint in patches, umber (13i) (reverse); chlamydospores extensive 

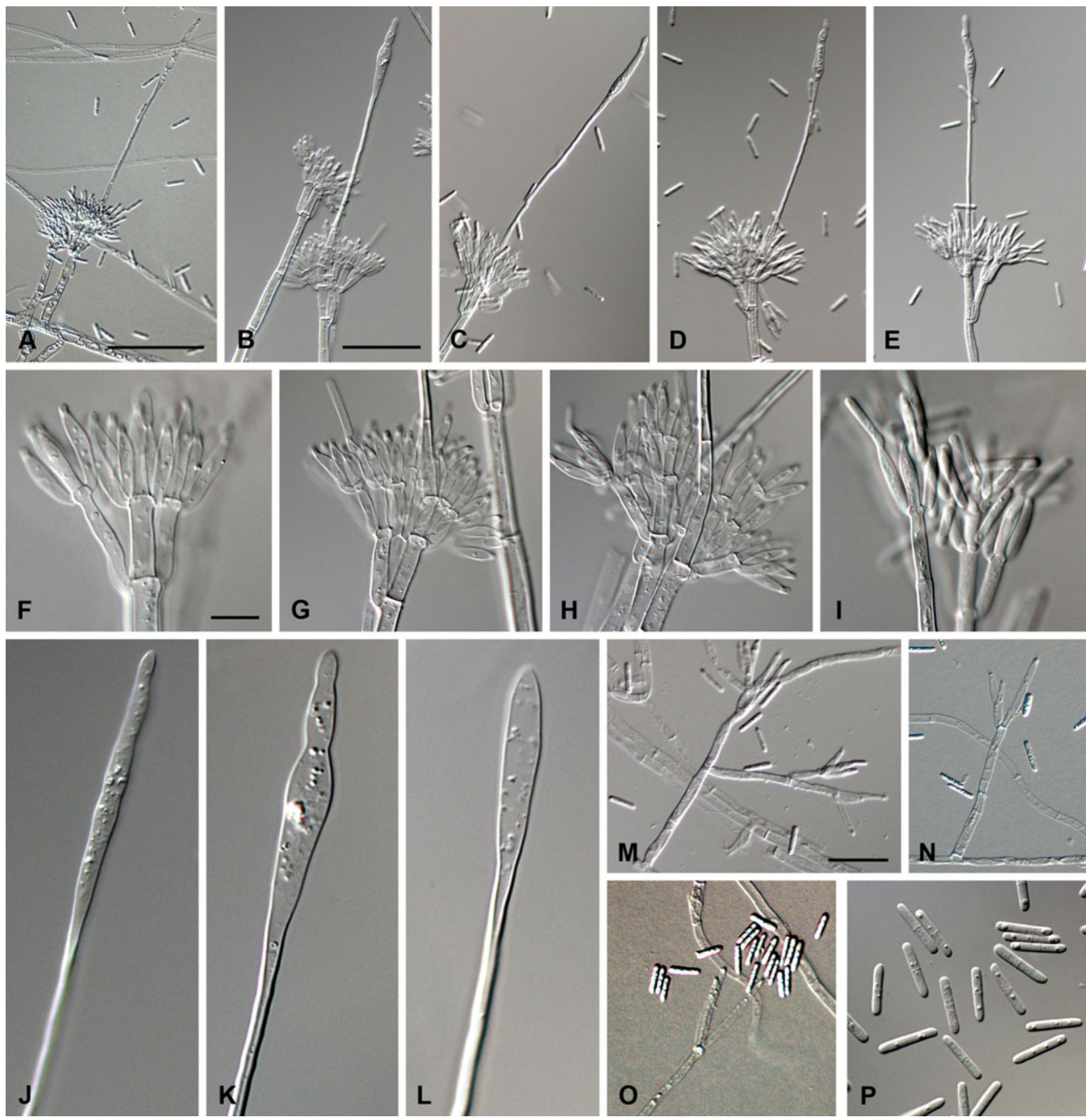

Fig. 7 Cylindrocladiella kurandica. a-e. Penicillate conidiophores. fi. Conidiogenous apparatus with conidiophore branches and phialides. $\mathbf{j}-\mathbf{l}$. Terminal vesicles. $\mathbf{m}-\mathbf{0}$. Subverticillate conidiophores. p. Conidia.

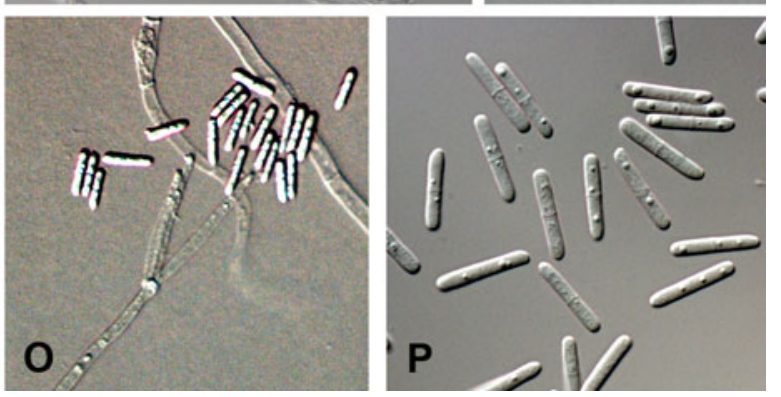

Scale bars: $\mathrm{A}=50 \mu \mathrm{m}, \mathrm{B}=20 \mu \mathrm{m}$ (apply to $\mathbf{c}-\mathbf{e}, \mathbf{m}-\mathbf{0}), \mathrm{F}=10 \mu \mathrm{m}$ (apply to $\mathbf{g}-\mathbf{l}, \mathbf{p}$ )

throughout medium, arranged in chains; reaching $65 \mathrm{~mm}$ after 7 days on MEA at $24^{\circ} \mathrm{C}$ in the dark.

Specimen examined - Australia, Queensland, Kuranda, from soil, 13 Aug 2009, P.W. Crous, holotype CBSH20601, culture ex-type CBS 129577=CPC 17551; Australia, Queensland, Kuranda, from soil, 13 Aug 2009, P.W. Crous, culture CBS 129576=CPC 17547; Australia,
Queensland, Kuranda, from soil, 13 Aug 2009, P.W. Crous, culture CPC 17549.

Notes - Cylindrocladiella kurandica is difficult to distinguish from $C$. longistipitata and other species in the $C$. infestans complex, and therefore phylogenetic inference is required for an accurate identification. Cylindrocladiella kurandica can be distinguished from other species in the $C$. infestans 
complex by different unique fixed nucleotides for three loci: BTUB positions 97 (T), 395 (A) and 482 (T); HIS3 positions $22(\mathrm{~T}), 50(\mathrm{~A})$ and $315(\mathrm{~T})$; TEF-1 $\alpha$ position $107(\mathrm{C})$.

Cylindrocladiella lanceolata L. Lombard \& Crous, sp. nov. - MycoBank MB561675, Fig. 8.

Etymology - Named after the lanceolate shape of its vesicles.

Cylindrocladiellae lageniformis morphologice similis, sed vesiculis lanceolatis distinguitur.

Teleomorph unknown. Conidiophores monomorphic, penicillate, mononematous and hyaline. Penicillate conidiophores (Fig. 8a-e) comprising a stipe, a penicillate arrangement of fertile branches, a stipe extension and a terminal vesicle; stipe septate, hyaline, smooth, 31-77× 5-10 $\mu \mathrm{m}$; stipe extension aseptate, straight, $76-173 \mu \mathrm{m}$ long, thick-walled with one basal septum, terminating in thin-walled, lanceolate vesicles (Fig. $8 \mathrm{f}-\mathrm{h}$ ), 5-7 $\mu \mathrm{m}$ wide. Penicillate conidiogenous apparatus (Fig. 8i-k) with primary branches aseptate, $12-30 \times 3-8 \mu \mathrm{m}$, secondary branches aseptate, $7-17 \times 3-6 \mu \mathrm{m}$, each terminal branch producing 2-4 phialides; phialides reniform to doliiform to cymbiform, hyaline, aseptate, 7-13 $\times 2$ $3 \mu \mathrm{m}$, apex with minute periclinal thickening and collarette absent. Subverticillate conidiophores not observed. Conidia (Fig. 81) cylindrical, rounded at both ends, straight, 1-septate, $(13-) 15-17(-20) \times 2-3 \mu \mathrm{m}($ av. $=16 \times 3 \mu \mathrm{m})$, frequently slightly flattened at the base, held in asymmetrical clusters by colourless slime.

Culture characteristics - Colonies raised (convex), cottony, with smooth margins, white, umber (13i) (reverse); chlamydospores extensive throughout medium, arranged in chains; reaching $55 \mathrm{~mm}$ after 7 days on MEA at $24^{\circ} \mathrm{C}$ in the dark.

Specimens examined - Australia, Queensland, Brisbane, from soil, 18 July 2009, P.W. Crous, holotype CBS-H20602, culture ex-type CBS 129566=CPC 17567, CBS 129565= CPC 17566; South Africa, KwaZulu-Natal, Kwambonambi, Mondi Sawmill, from Eucalyptus sp., 1 May 1990, P.W. Crous, culture CBS 114950=CPC 396.
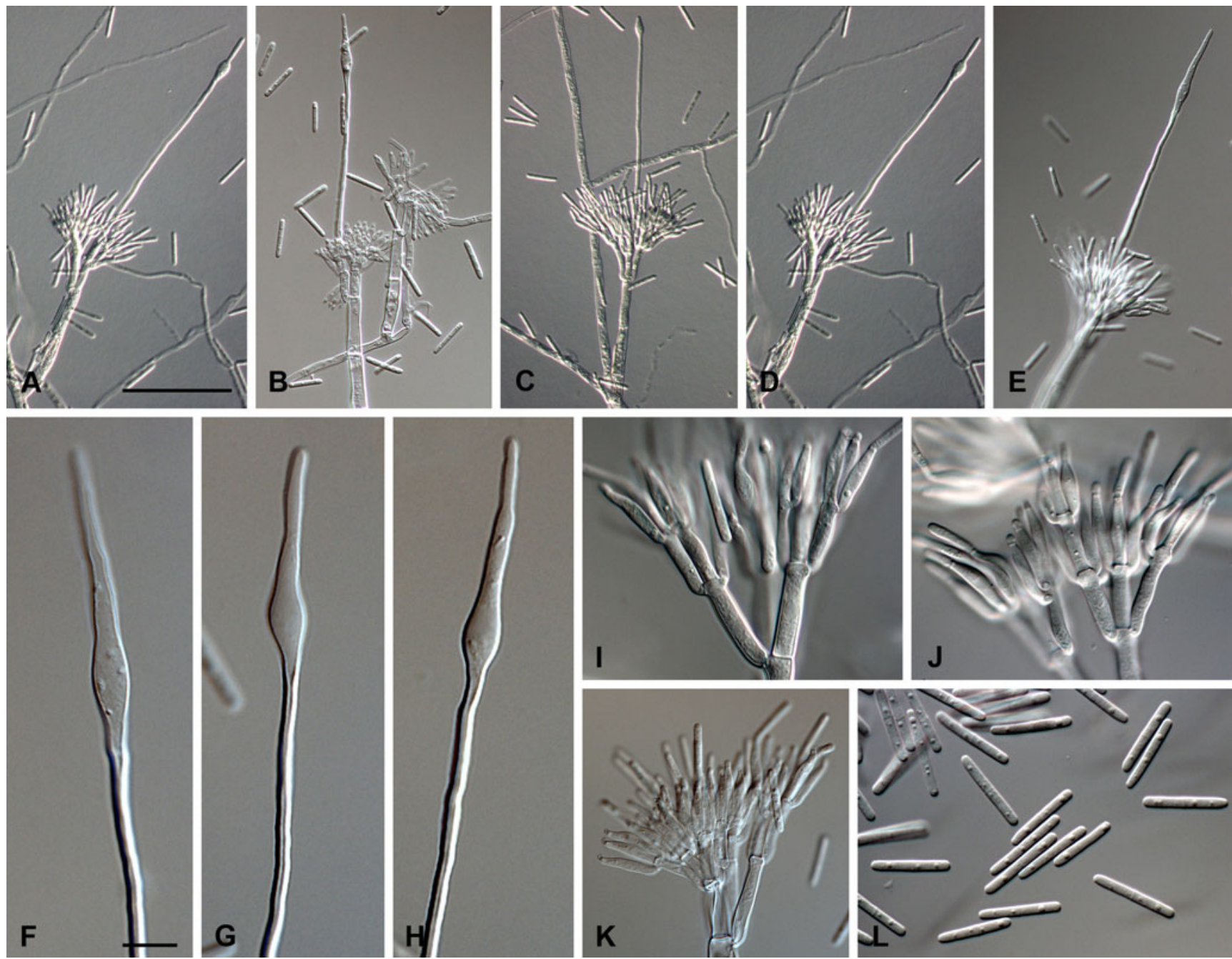

Fig. 8 Cylindrocladiella lanceolata. a-e. Penicillate conidiophores. $\mathbf{f}-\mathbf{h}$. Terminal vesicles. $\mathbf{i}-\mathbf{k}$. Conidiogenous apparatus with conidiophore branches and phialides. L. Conidia. Scale bars: $A=50 \mu \mathrm{m}$ (apply to $\mathbf{b}-\mathbf{e}$ ), $\mathrm{F}=10 \mu \mathrm{m}$ (apply to $\mathbf{g}-\mathbf{I}$ ) 
Note-Cylindrocladiella lanceolata can be distinguished from $C$. lageniformis by its lanceolate terminal vesicles and conidium dimensions.

Cylindrocladiella longiphialidica L. Lombard \& Crous, sp. nov. - MycoBank MB561669, Fig. 9.
Etymology - Named after its characteristically long phialides.

Cylindrocladiellae camelliae morphologice valde similis, sed phialidibus conidiophorum subverticillatorum longioribus distinguitur.
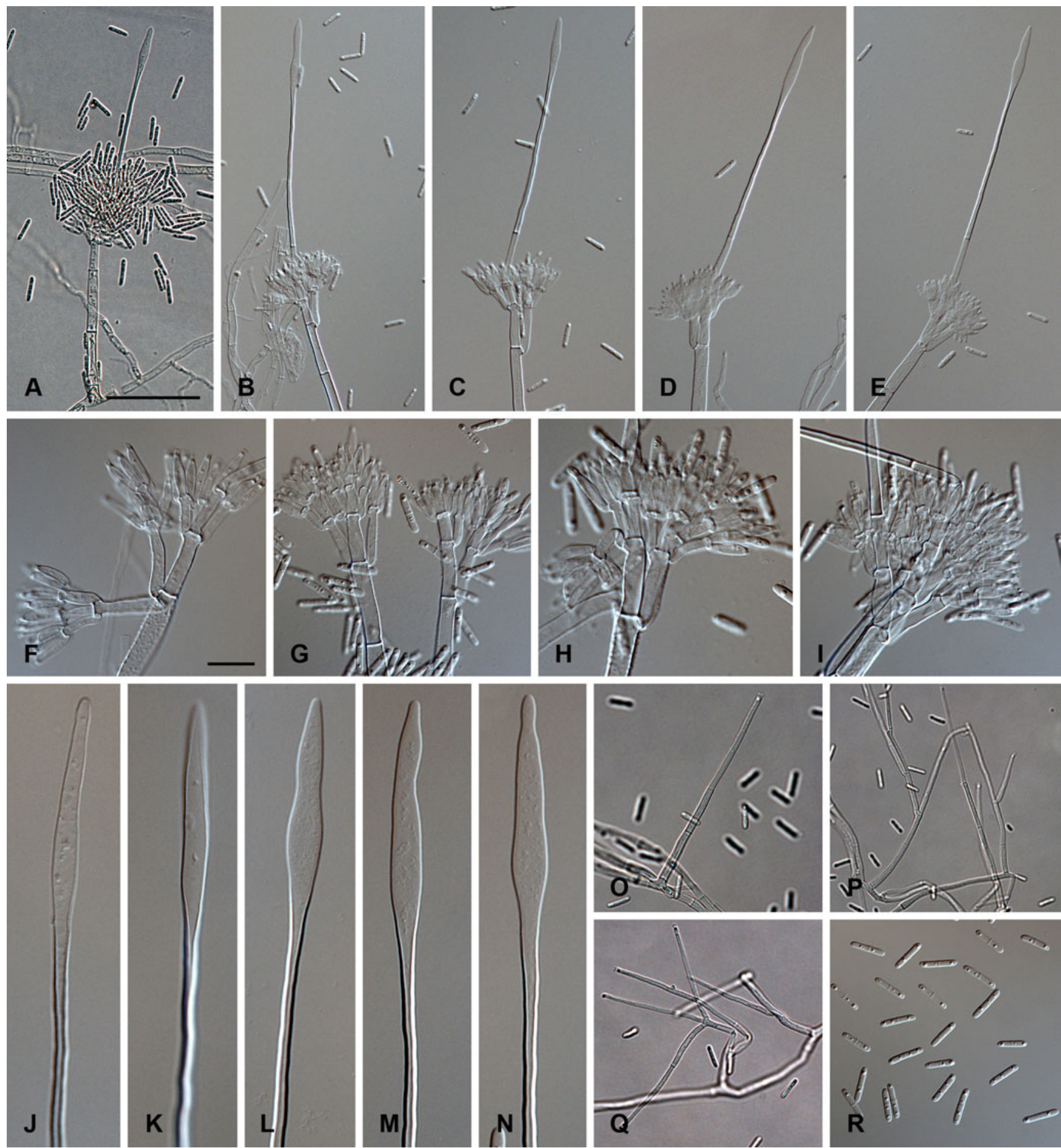

Fig. 9 Cylindrocladiella longiphialidica. a-e. Penicillate conidiophores. $\mathbf{f}-\mathbf{i}$. Conidiogenous apparatus with conidiophore branches and phialides. j-n. Terminal vesicles. o-q. Subverticillate conidiophores. R. Conidia. Scale bars: A=50 $\mu \mathrm{m}$ (apply to $\mathbf{b}-\mathbf{e}), \mathrm{F}=10 \mu \mathrm{m}$ (apply to $\mathbf{g}-\mathbf{r})$ 
Teleomorph unknown. Conidiophores dimorphic, penicillate and subverticillate, mononematous and hyaline. Penicillate conidiophores (Fig. 9a-e) comprising a stipe, a penicillate arrangement of fertile branches, a stipe extension and a terminal vesicle; stipe septate, hyaline, smooth, 43107×6-9 $\mu \mathrm{m}$; stipe extension aseptate, straight, 114$189 \mu \mathrm{m}$ long, thick-walled with one basal septum, terminating in thin-walled, ellipsoidal to lanceolate vesicles (Fig. 9j-n), 5$8 \mu \mathrm{m}$ wide. Penicillate conidiogenous apparatus (Fig. 9f-i) with primary branches aseptate, $11-33 \times 3-7 \mu \mathrm{m}$, secondary branches aseptate, 9-26×3-5 $\mu \mathrm{m}$, with each terminal branch producing 2-4 phialides; phialides doliiform to reniform to cymbiform, hyaline, aseptate, $8-13 \times 2-4 \mu \mathrm{m}$, apex with minute periclinal thickening and collarette. Subverticillate conidiophores (Fig. 9o-q) abundant, comprising of a septate stipe, and primary branches terminating in 1-3 phialides; primary branches straight, hyaline, 0-1-septate, 28-68×4 $6 \mu \mathrm{m}$; phialides cymbiform to cylindrical, hyaline, aseptate, $20-79 \times 2-5 \mu \mathrm{m}$, apex with minute periclinal thickening and collarette. Conidia (Fig. 9r) cylindrical, rounded at both ends, straight, 1-septate, $12-14 \times 2-3 \mu \mathrm{m}(\mathrm{av} .=13 \times$ $3 \mu \mathrm{m})$, frequently slightly flattened at the base, held in asymmetrical clusters by colourless slime.

Culture characteristics - Colonies raised (convex), cottony, with undulate margins, white centre becoming buff yellow (19 d) towards the margins, buff yellow (19 d) (reverse); chlamydospores extensive throughout medium, arranged in chains; reaching $55 \mathrm{~mm}$ after 7 days on MEA at $24^{\circ} \mathrm{C}$ in the dark.

Specimens examined - Thailand, Chiang Mai, from soil, Oct. 2010, P.W. Crous, holotype CBS-H20603, culture ex-type CBS 129557=CPC 18839; Thailand, Chiang Mai, from soil, Oct. 2010, P.W. Crous culture, CBS 129558= CPC 18841.

Notes - Cylindrocladiella longiphialidica is morphologically similar to C. nederlandica, C. pseudocamelliae and C. camelliae, but can be distinguished from these species by its longer phialides on the subverticillate conidiophores.

Cylindrocladiella longistipitata L. Lombard \& Crous, sp. nov. - MycoBank MB561679, Fig. 10.

Etymology - Named after its characteristically long stipe extensions on its conidiophores.

Cylindrocladiellae infestantis morphologice similis, sed extensionibus stipitis longioribus.

Teleomorph unknown. Conidiophores dimorphic, penicillate and subverticillate, mononematous and hyaline. Penicillate conidiophores (Fig. 10a-e) comprising a stipe, a penicillate arrangement of fertile branches, a stipe extension and a terminal vesicle; stipe septate, hyaline, smooth, 54-80 $\times 5-9 \mu \mathrm{m}$; stipe extension aseptate, straight, 130$216 \mu \mathrm{m}$ long, thick-walled with one basal septum, terminating in thin-walled, cylindrical to lanceolate vesicles (Fig. 10j-n), 5-7 $\mu \mathrm{m}$ wide. Penicillate conidiogenous apparatus
(Fig. 10f-i) with primary branches aseptate, 13-20 $\times 3-$ $5 \mu \mathrm{m}$, secondary branches aseptate, 9-13 $\times 3-5 \mu \mathrm{m}$, each terminal branch producing 2-4 phialides; phialides cymbiform to cylindrical, hyaline, aseptate, $10-16 \times 2-4 \mu \mathrm{m}$, apex with minute periclinal thickening and collarette. Subverticillate conidiophores (Fig. 10o-p) in moderate numbers, comprising of a septate stipe, and primary branches terminating in 2-3 phialides; primary branches straight, hyaline, 0-1-septate, 21-40×4 $\mu \mathrm{m}$; phialides cymbiform to cylindrical, hyaline, aseptate, $18-31 \times 2-$ $4 \mu \mathrm{m}$, apex with minute periclinal thickening and collarette. Conidia (Fig. 10q) cylindrical, rounded at both ends, straight, 1-septate, $(12-) 14-16(-17) \times 2-4 \mu \mathrm{m}$ (av. = $15 \times 3 \mu \mathrm{m})$, frequently slightly flattened at the base, held in asymmetrical clusters by colourless slime.

Culture characteristics - Colonies raised (convex), cottony, with smooth to undulate margins, white, umber (13i) (reverse); chlamydospores extensive throughout medium, arranged in chains; reaching $45 \mathrm{~mm}$ after 7 days on MEA at $24^{\circ} \mathrm{C}$ in the dark.

Specimens examined - China, Hong Kong, from soil, Nov. 1993, M.J. Wingfield, holotype CBS-H20604, culture ex-type CBS 116075=CPC 708; Australia, Queensland, Topaz, Atherton Tablelands, from Opisthiolepsis heterophylla, 2 Apr. 2001, C. Pearce \& B. Paulus, culture CBS $112953=$ CPC 4720.

Notes - Cylindrocladiella longistipitata can be distinguished from other species in the $C$. infestans complex by its longer stipe extension and terminal vesicle morphology. Furthermore, it has unique fixed nucleotides for three loci: BTUB position $363(\mathrm{~A})$; HIS3 positions $37(\mathrm{C})$ and $400(\mathrm{~T})$; TEF-1 $\alpha$ positions $44(\mathrm{~A})$ and 45 (T).

Cylindrocladiella natalensis L. Lombard \& Crous, sp. nov. - MycoBank MB561670, Fig. 11.

Etymology - Named after the Province in South Africa where this fungus was first collected, KwaZulu-Natal.

Cylindrocladiellae elegantis morphologice valde similis, sed conidiis majoribus, (12-)14-16(-17) $\times 2-3 \mu \mathrm{m}$, distinguitur.

Teleomorph unknown. Conidiophores dimorphic, penicillate and subverticillate, mononematous and hyaline. Penicillate conidiophores (Fig. 11a-e) comprising a stipe, a penicillate arrangement of fertile branches, a stipe extension and a terminal vesicle; stipe septate, hyaline, smooth, 88-135 $\times 5-8 \mu \mathrm{m}$; stipe extension aseptate, straight, 82$127 \mu \mathrm{m}$ long, thick-walled with one basal septum, terminating in thin-walled, ellipsoidal to fusoid vesicles (Fig. $11 \mathrm{j}-\mathrm{m}$ ), 6 $8 \mu \mathrm{m}$ wide. Penicillate conidiogenous apparatus (Fig. 11f-i) with primary branches aseptate, $13-29 \times 2-5 \mu \mathrm{m}$, secondary branches aseptate, 8-17×3-4 $\mu \mathrm{m}$, each terminal branch producing 2-4 phialides; phialides cymbiform to cylindrical, hyaline, aseptate, $9-14 \times 2-3 \mu \mathrm{m}$, apex with minute periclinal thickening and collarette. Subverticillate conidiophores 

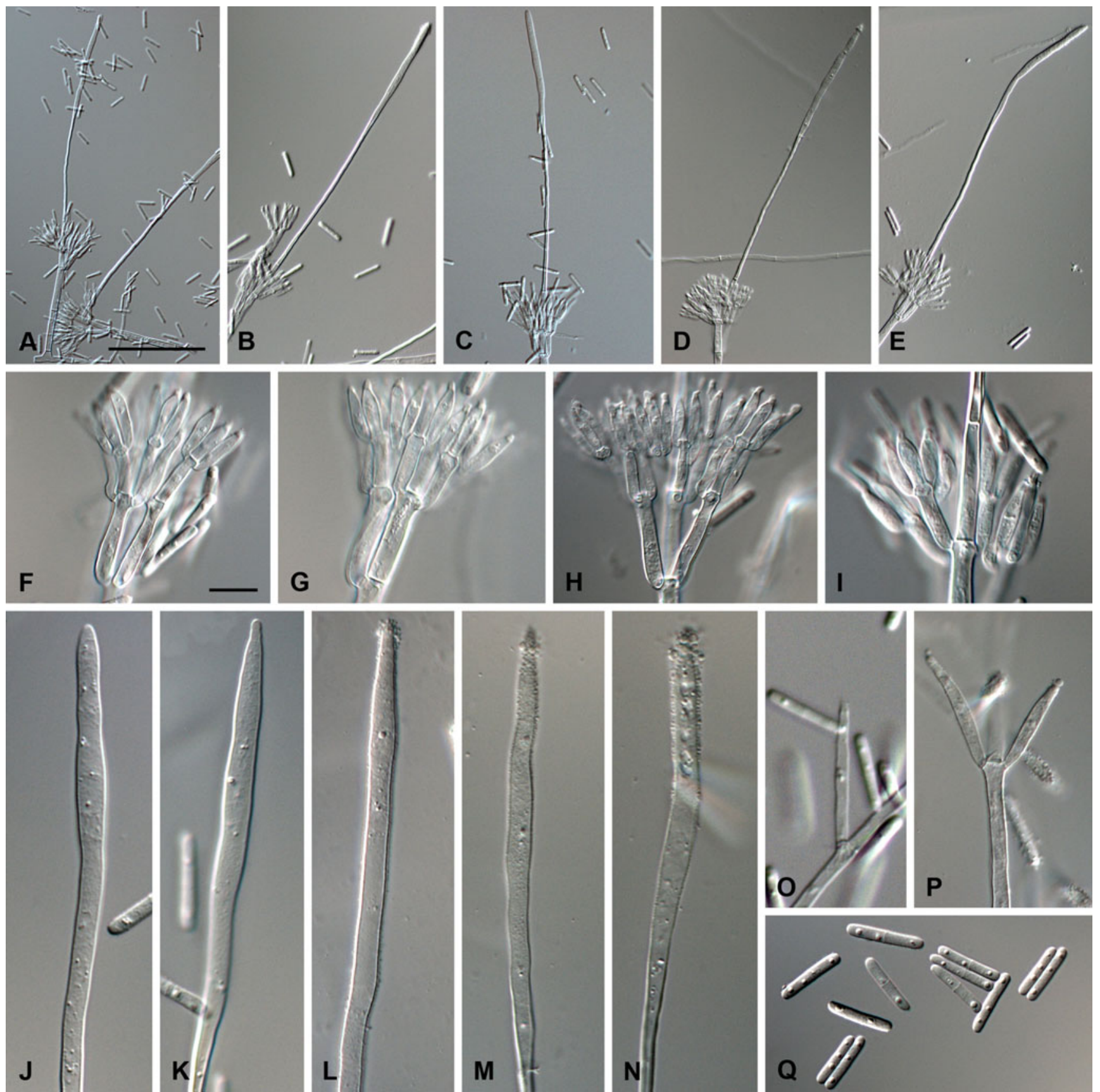

Fig. 10 Cylindrocladiella longistipitata. a-e. Penicillate conidiophores. $\mathbf{f}-\mathbf{i}$. Conidiogenous apparatus with conidiophore branches and phialides. $\mathbf{j}-\mathbf{n}$. Terminal vesicles. o-p. Subverticillate conidiophores. Q. Conidia. A=50 $\mu \mathrm{m}$ (apply to $\mathbf{b}-\mathbf{e}), \mathrm{F}=10 \mu \mathrm{m}$ (apply to $\mathbf{g}-\mathbf{q})$

(Fig. 11n-0) in moderate numbers, comprising of a septate stipe, and primary branches terminating in 2-3 phialides; primary branches straight, hyaline, $0-1$-septate, $23-39 \times 2-$ $4 \mu \mathrm{m}$; phialides cymbiform to cylindrical, hyaline, aseptate, 19-34×2-4 $\mu \mathrm{m}$, apex with minute periclinal thickening and collarette. Conidia (Fig. 11p) cylindrical, rounded at both ends, straight, 1-septate, $(12-) 14-16(-17) \times 2-3 \mu \mathrm{m}$ (av. = $15 \times 3 \mu \mathrm{m})$, frequently slightly flattened at the base, held in asymmetrical clusters by colourless slime.
Culture characteristics - Colonies raised (convex), cottony, with smooth to undulate margins, white, buff yellow (19 d) (reverse); chlamydospores sparse throughout medium, arranged in chains; reaching $70 \mathrm{~mm}$ after 7 days on MEA at $24^{\circ} \mathrm{C}$ in the dark.

Specimens examined - South Africa, KwaZulu-Natal, from Arachis hypogaea, 1 Feb. 1991, M.J. Wingfield, holotype CBS-H20605, culture ex-type CBS 114943= CPC 456, CBS 114945=CPC 459; Australia, Queensland, 

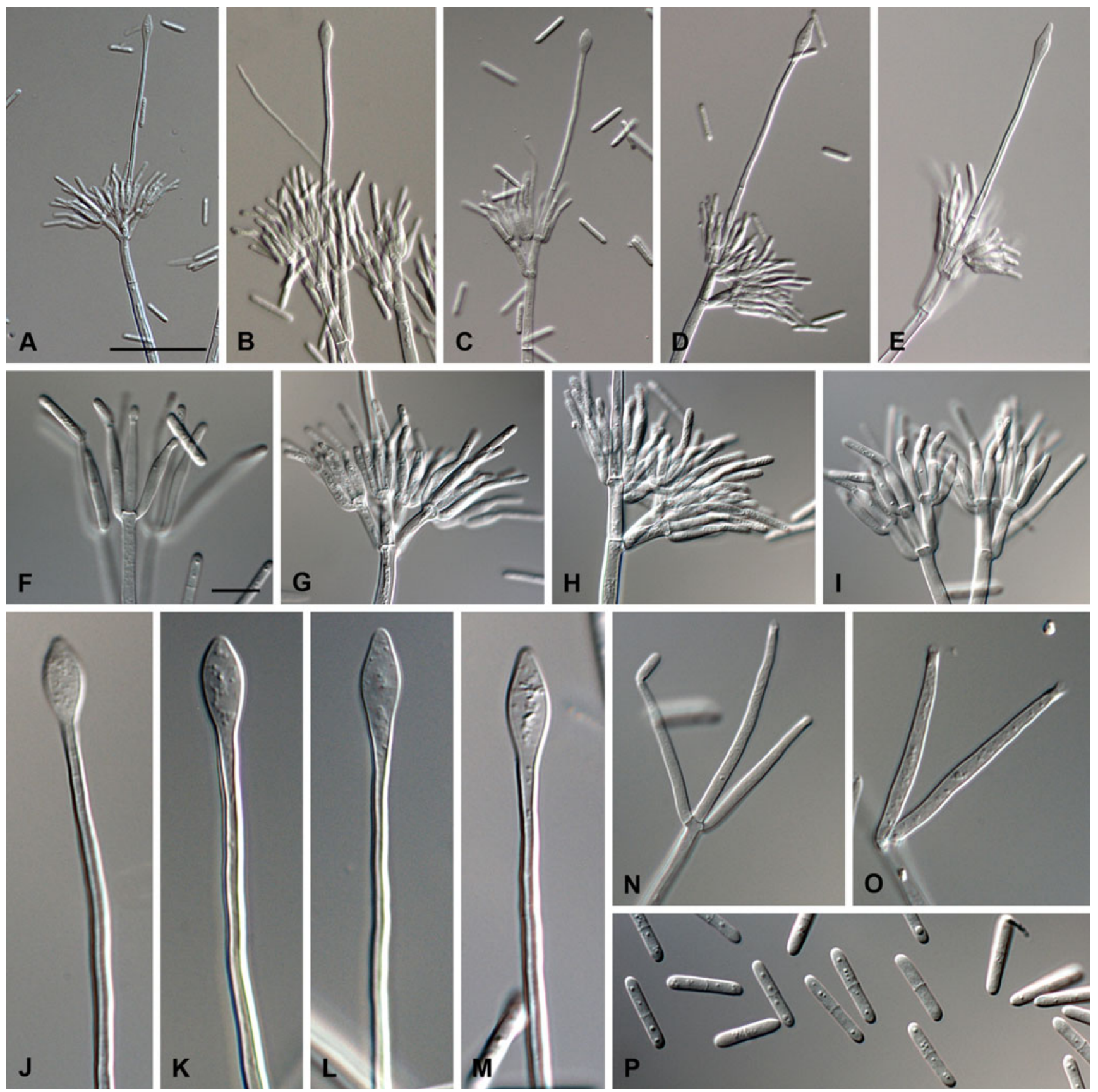

Fig. 11 Cylindrocladiella natalensis. a-e. Penicillate conidiophores. $\mathbf{f}-\mathbf{i}$. Conidiogenous apparatus with conidiophore branches and phialides. $\mathbf{j}-\mathbf{m}$. Terminal vesicles. $\mathbf{n}-\mathbf{o}$. Subverticillate conidiophores. P. Conidia. Scale bars: A=50 $\mu \mathrm{m}$ (apply to $\mathbf{b}-\mathbf{e}), \mathrm{F}=10 \mu \mathrm{m}$ (apply to $\mathbf{g}-\mathbf{p})$

Byron Bay, from soil, 17 July 2009, P.W. Crous, culture CPC 17395.

Note - Cylindrocladiella natalensis can be distinguished from other species in this genus by its conidium dimensions and shape of the terminal vesicle.

Cylindrocladiella nederlandica L. Lombard \& Crous, sp. nov. - MycoBank MB561667, Fig. 12.

Etymology - Named after the Netherlands, the country where this fungus was collected.
Cylindrocladiellae camelliae morphologice valde similis, sed phialidibus majoribus, $14-30 \times 3-5 \mu \mathrm{m}$, distinguitur.

Teleomorph unknown. Conidiophores dimorphic, penicillate and subverticillate, mononematous and hyaline. Penicillate conidiophores (Fig. 12a-e) comprising a stipe, a penicillate arrangement of fertile branches, a stipe extension and a terminal vesicle; stipe septate, hyaline, smooth, 41-124×4-10 $\mu \mathrm{m}$; stipe extension aseptate, straight, 102 $158 \mu \mathrm{m}$ long, thick-walled with one basal septum, 

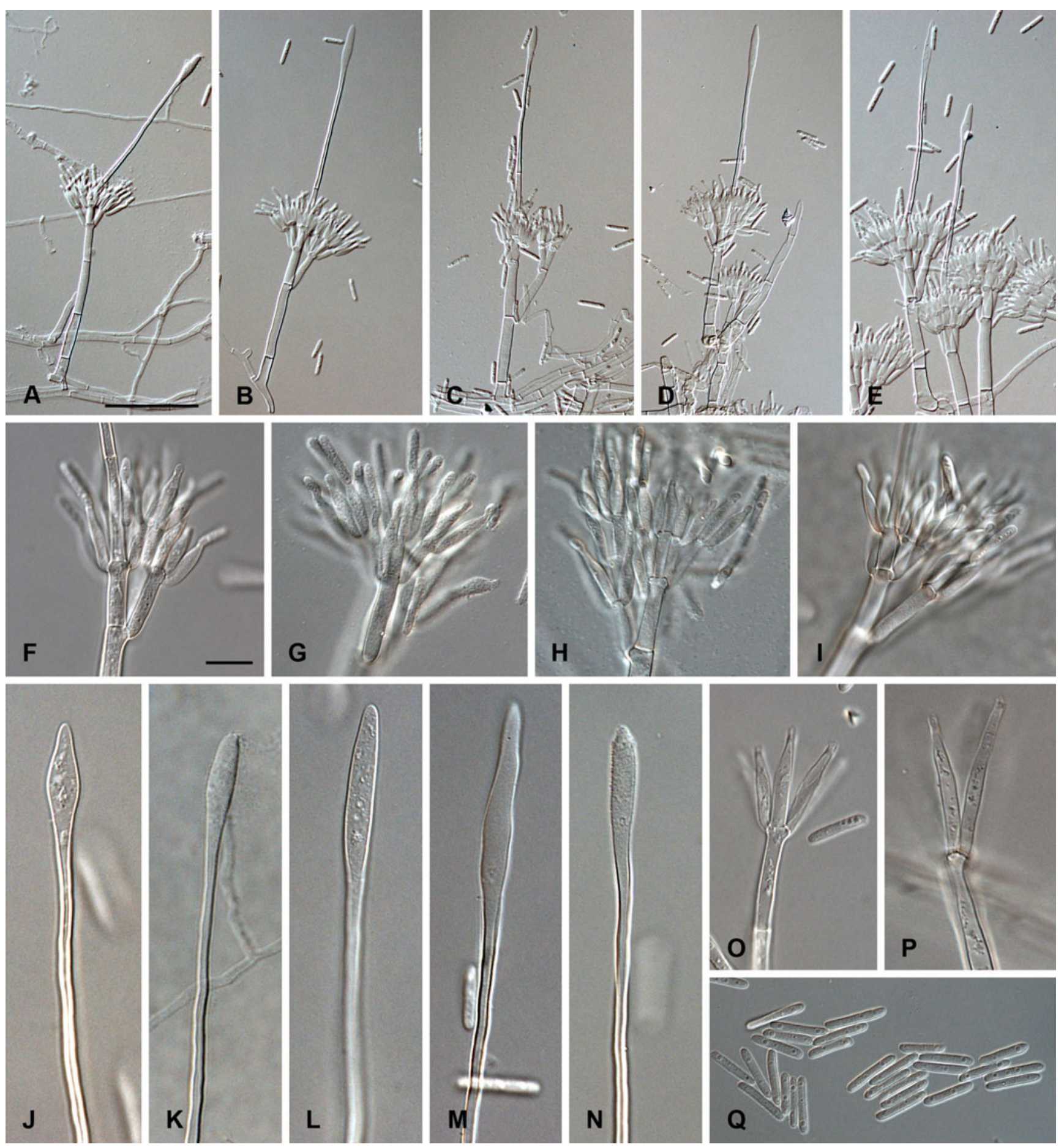

Fig. 12 Cylindrocladiella nederlandica. a-e. Penicillate conidiophores. $\mathbf{f}-\mathbf{i}$. Conidiogenous apparatus with conidiophore branches and phialides. $\mathbf{j}-\mathbf{n}$. Terminal vesicles. o-p. Subverticillate conidiophores. Q. Conidia. Scale bars: A=50 $\mu \mathrm{m}$ (apply to $\mathbf{b}-\mathbf{e}$ ), $\mathrm{F}=10 \mu \mathrm{m}($ apply to $\mathbf{g}-\mathbf{q})$

terminating in thin-walled, lageniform to ellipsoidal vesicles (Fig. 12j-n), 4-9 $\mu \mathrm{m}$ wide. Penicillate conidiogenous apparatus (Fig. 12f-i) with primary branches aseptate, $12-31 \times 3-7 \mu \mathrm{m}$, secondary branches aseptate, $8-18 \times 2-$ $5 \mu \mathrm{m}$, each terminal branch producing 2-4 phialides; phialides doliiform to reniform to cymbiform, hyaline, aseptate, $8-14 \times 2-4 \mu \mathrm{m}$, apex with minute periclinal thickening and collarette. Subverticillate conidiophores (Fig. 12o-p) abundant, comprising of a septate stipe, and primary branches terminating in 1-3 phialides; primary branches straight, hyaline, $0-1$-septate, $18-32 \times 3-5 \mu \mathrm{m}$; phialides cymbiform to cylindrical, hyaline, aseptate, 14-30 $\times 3-5 \mu \mathrm{m}$, apex with minute periclinal thickening and collarette. Conidia (Fig. 12q) cylindrical, rounded at 
both ends, straight, 1-septate, $(10-) 12-14(-15) \times 2-4 \mu \mathrm{m}$ (av. $=13 \times 2 \mu \mathrm{m})$, frequently slightly flattened at the base, held in asymmetrical clusters by colourless slime.

Culture characteristics - Colonies raised (convex), cottony, with smooth to undulate margins, white, buff yellow (19 d) to umber (13i) (reverse); chlamydospores moderate throughout medium, arranged in chains; reaching $55 \mathrm{~mm}$ after 7 days on MEA at $24^{\circ} \mathrm{C}$ in the dark.

Specimens examined - The Netherlands, from Pelargonium sp., Mar. 1991, J.W. Veenbaas-Rijks, holotype CBS-H5129, culture ex-type CBS 152.91=PD 90/2015; The Netherlands, Aalsmeer, from Kalanchoë sp., Feb. 1995, J.W. Veenbaas-Rijks, culture CBS 143.95=PD 94/1353; The Netherlands, stem of Rhododendron, Mar. 1994, culture CBS 146.94=PD 39/1776.

Notes - Morphologically, isolates of C. nederlandica are very similar to $C$. camelliae, with a slight difference in terminal vesicle shape. The phialides on the subverticillate conidiophores of $C$. nederlandica $(14-30 \times 3-5 \mu \mathrm{m})$ are larger than those of $C$. camelliae $(15-26 \times 2-3.5 \mu \mathrm{m}$; Crous 2002).

Cylindrocladiella pseudocamelliae L. Lombard \& Crous, sp. nov. - MycoBank MB561668, Fig. 13.

Etymology - Named after its morphological similarity to Cylindrocladiella camelliae.

Cylindrocladiellae camelliae morphologice similis, sed vesiculis divergentibus.

Teleomorph unknown. Conidiophores dimorphic, penicillate and subverticillate, mononematous and hyaline. Penicillate conidiophores (Fig. 13a-e) comprising a stipe, a penicillate arrangement of fertile branches, a stipe extension and a terminal vesicle; stipe septate, hyaline, smooth, 65-137×6-10 $\mu \mathrm{m}$; stipe extension aseptate, straight, 106$188 \mu \mathrm{m}$ long, thick-walled with one basal septum, terminating in thin-walled, ellipsoidal to lageniform to lanceolate vesicles (Fig. 13j-n), 6-10 $\mu \mathrm{m}$ wide. Penicillate conidiogenous apparatus (Fig. 13f-i) with primary branches aseptate, $12-27 \times 3-6 \mu \mathrm{m}$, secondary branches aseptate, $8-18 \times 2-$ $5 \mu \mathrm{m}$, each terminal branch producing 2-4 phialides; phialides doliiform to reniform to cymbiform, hyaline, aseptate, $10-17 \times 2-3 \mu \mathrm{m}$, apex with minute periclinal thickening and collarette. Subverticillate conidiophores (Fig. 13o-p) abundant, comprising of a septate stipe, and primary branches terminating in 1-3 phialides; primary branches straight, hyaline, 0-1-septate, 15-32×3-6 $\mu \mathrm{m}$; phialides cymbiform to cylindrical, hyaline, aseptate, $19-31 \times 3-5 \mu \mathrm{m}$, apex with minute periclinal thickening and collarette. Conidia (Fig. 13q) cylindrical, rounded at both ends, straight, 1-septate, (9-)11-15(-16) $\times 2-4 \mu \mathrm{m}$ $($ av. $=13 \times 3 \mu \mathrm{m})$, frequently slightly flattened at the base, held in asymmetrical clusters by colourless slime.

Culture characteristics - Colonies raised (convex), cottony, with smooth margins, white, buff yellow (19 d) to umber (13i) (reverse); chlamydospores moderate throughout medium, arranged in chains; reaching $90 \mathrm{~mm}$ after 7 days on MEA at $24^{\circ} \mathrm{C}$ in the dark.

Specimens examined - Thailand, Chiang Mai, from soil, Oct. 2010, P.W. Crous, holotype CBS-H20606, culture ex-type CBS 129555=CPC 18825; Thailand, Chiang Mai, from soil, Oct. 2010, P.W. Crous, culture CBS 129556=CPC 18832; Thailand, Chiang Mai, from soil, Oct. 2010, P.W. Crous, culture CPC 18838.

Notes - As with C. nederlandica, C. pseudocamelliae is morphologically similar to C. camelliae. However, C. pseudocamelliae can be distinguished from both the other species by its longer stipe extension and the shape of its terminal vesicle.

Cylindrocladiella pseudohawaiiensis L. Lombard \& Crous, sp. nov. - MycoBank MB561678, Fig. 14.

Etymology - Named after its morphological similarity to Cylindrocladiella hawaiiensis.

Cylindrocladiellae infestantis morphologice valde similis, sed conidiis minoribus, $(11-) 12-14(-15) \times 2-4 \mu \mathrm{m}$, distinguitur.

Teleomorph unknown. Conidiophores dimorphic, penicillate and subverticillate, mononematous and hyaline. Penicillate conidiophores (Fig. 14a-c) comprising a stipe, a penicillate arrangement of fertile branches, a stipe extension and a terminal vesicle; stipe septate, hyaline, smooth, 31-62× 5-8 $\mu \mathrm{m}$; stipe extension aseptate, straight, 70-97 $\mu \mathrm{m}$ long, thick-walled with one basal septum, terminating in thin-walled, clavate to ellipsoidal vesicles (Fig. $14 \mathrm{~d}-$ f), 6-8 $\mu \mathrm{m}$ wide. Penicillate conidiogenous apparatus (Fig. 14g-i) with primary branches aseptate, $9-19 \times 3-5 \mu \mathrm{m}$, secondary branches aseptate, $9-11 \times 4 \mu \mathrm{m}$, each terminal branch producing $2-4$ phialides; phialides cymbiform to cylindrical, hyaline, aseptate, $10-15 \times 2-4 \mu \mathrm{m}$, apex with minute periclinal thickening and collarette. Subverticillate conidiophores (Fig. 14j-k) in moderate numbers, comprising of a septate stipe, and primary branches terminating in 2-3 phialides; primary branches straight, hyaline, $0-1$-septate, 16$40 \times 4 \mu \mathrm{m}$; phialides cymbiform to cylindrical, hyaline, aseptate, $17-28 \times 3-4 \mu \mathrm{m}$, apex with minute periclinal thickening and collarette. Conidia (Fig. 141) cylindrical, rounded at both ends, straight, 1-septate, (11-)12-14(-15) $\times 2-4 \mu \mathrm{m}$ (av. = $13 \times 3 \mu \mathrm{m}$ ), frequently slightly flattened at the base, held in asymmetrical clusters by colourless slime.

Culture characteristics - Colonies raised (convex), cottony, with smooth margins, white, buff yellow (19 d) to umber (13i) (reverse); chlamydospores extensive throughout medium, arranged in chains; reaching $75 \mathrm{~mm}$ after 7 days on MEA at $24^{\circ} \mathrm{C}$ in the dark.

Specimens examined - Brazil, Sao Paulo, Aracruz nursery, from Eucalyptus cutting, 1992, A.C. Alfenas, holotype CBSH20607, culture ex-type CBS 210.94=PPRI 4450=UFV 125=IMI 361579; Madagascar, Isoamala-Beraketa, Mount 

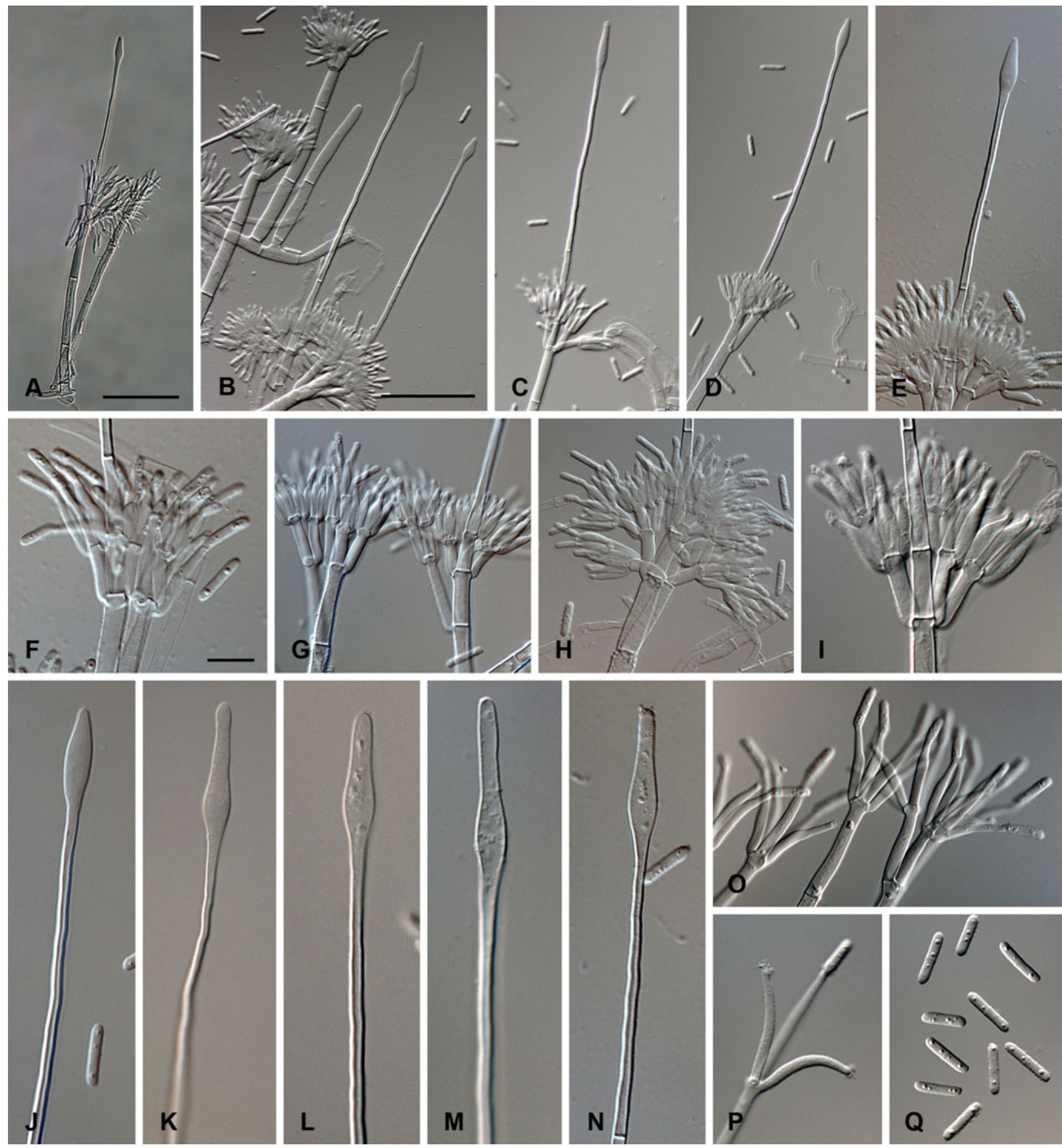

Fig. 13 Cylindrocladiella pseudocamelliae. a-e. Penicillate conidiophores. $\mathbf{f}-\mathbf{i}$. Conidiogenous apparatus with conidiophore branches and phialides. $\mathbf{j}-\mathbf{n}$. Terminal vesicles. $\mathbf{o}-\mathbf{p}$. Subverticillate conidiophores.

Q. Conidia. Scale bars: $\mathrm{A}=50 \mu \mathrm{m}, \mathrm{B}=50 \mu \mathrm{m}$ (apply to $\mathbf{c}-\mathbf{e}$ ), $\mathrm{F}=10 \mu \mathrm{m}$ (apply to $\mathbf{g - q}$ )

Tolongo, substrate unknown, 7 Mar. 1994, collector unknown, culture CBS 115610=CPC 909=Fox 409.

Notes - Morphologically C. pseudohawaiiensis is difficult to distinguish from $C$. hawaiiensis, and therefore phylogenetic inference is required. It can be distinguished from $C$. infestans by its smaller conidium dimensions and terminal vesicle

shape. Cylindrocladiella pseudohawaiiensis can also be distinguished from other species in the $C$. infestans complex by different unique fixed nucleotides for three loci: BTUB positions 127 (A) and $384(\mathrm{G})$; HIS3 positions 23 (C), 29 (C), 33 (A), 77 (G), 283 (indel), 285 (C), 288 (A), 314 (T), 349 (T) and 463 (T); TEF-1 $\alpha$ 

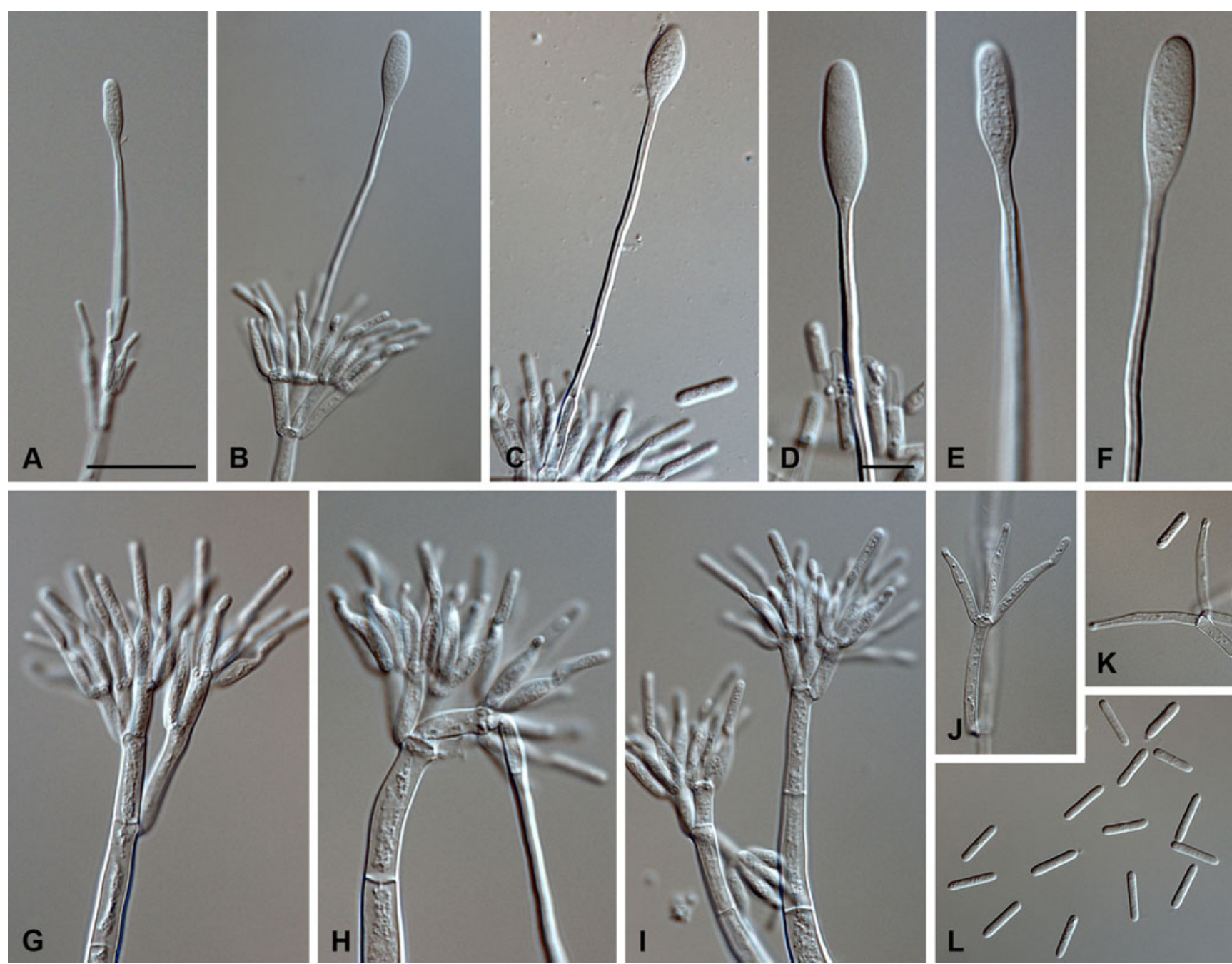

Fig. 14 Cylindrocladiella pseudohawaiiensis. a-c. Penicillate conidiophores. $\mathbf{d}-\mathbf{f}$. Terminal vesicles. $\mathbf{g}-\mathbf{i}$. Conidiogenous apparatus with conidiophore branches and phialides. $\mathbf{j}-\mathbf{k}$. Subverticillate conidiophores. L. Conidia. A=20 $\mu \mathrm{m}$ (apply to $\mathbf{b}-\mathbf{c}$ ), $\mathrm{D}=10 \mu \mathrm{m}$ (apply to $\mathbf{e}-\mathbf{l}$ )

positions $153(\mathrm{~T}), 244(\mathrm{~T}), 288(\mathrm{~T}), 289(\mathrm{~A}), 290(\mathrm{~T})$, $337(\mathrm{C}), 465(\mathrm{~A}), 471(\mathrm{G}), 478(\mathrm{~T})$ and $482(\mathrm{G})$.

Cylindrocladiella pseudoparva L. Lombard \& Crous, sp. nov. - MycoBank MB561672, Fig. 15.

Etymology - Named after its morphological similarity to Cylindrocladiella parva.

Cylindrocladiellae parvae morphologice valde similis, sed ramis primariis conidiophorum majoribus distinguitur.

Teleomorph unknown. Conidiophores monomorphic, penicillate, mononematous and hyaline. Penicillate conidiophores (Fig. 15a-e) comprising a stipe, a penicillate arrangement of fertile branches, a stipe extension and a terminal vesicle; stipe septate, hyaline, smooth, 31-86×5-9 $\mu \mathrm{m}$; stipe extension aseptate, straight, 111-164 $\mu \mathrm{m}$ long, thick-walled with one basal septum, terminating in thin-walled, clavate to ellipsoidal to pyriform vesicles (Fig. 15f-h), 5-7 $\mu \mathrm{m}$ wide. Penicillate conidiogenous apparatus (Fig.15i-k) with primary branches aseptate, 16-32 $\times 3-6 \mu \mathrm{m}$, secondary branches aseptate, $8-18 \times 3-$
$5 \mu \mathrm{m}$, each terminal branch producing 2-4 phialides; phialides doliiform to cymbiform, hyaline, aseptate, 10 $17 \times 2-4 \mu \mathrm{m}$, apex with minute periclinal thickening and collarette absent. Subverticillate conidiophores not observed. Conidia (Fig. 151) cylindrical, rounded at both ends, straight, 1-septate, 16-18(-20) $\times 2-4 \mu \mathrm{m}(\mathrm{av} .=17 \times$ $3 \mu \mathrm{m}$ ), frequently slightly flattened at the base, held in asymmetrical clusters by colourless slime.

Culture characteristics - Colonies raised (convex), cottony, with smooth margins, white with buff yellow (19 d) centre, umber (13i) (reverse); chlamydospores extensive throughout medium, arranged in chains; reaching $50 \mathrm{~mm}$ after 7 days on MEA at $24^{\circ} \mathrm{C}$ in the dark.

Specimens examined - The Netherlands, Apeldoorn, Paleis Het Loo, from soil, Apr. 2010, P.W. Crous, holotype CBS-H20608, culture ex-type CBS $129560=$ CPC 18149; New Zealand, South Auckland, Karaka, Karaka road, from Vitis riparia, 16 Apr. 2007, K. Paice, culture CBS 122594; Switzerland, Mohlin Canton, Basel, from root of Quercus 

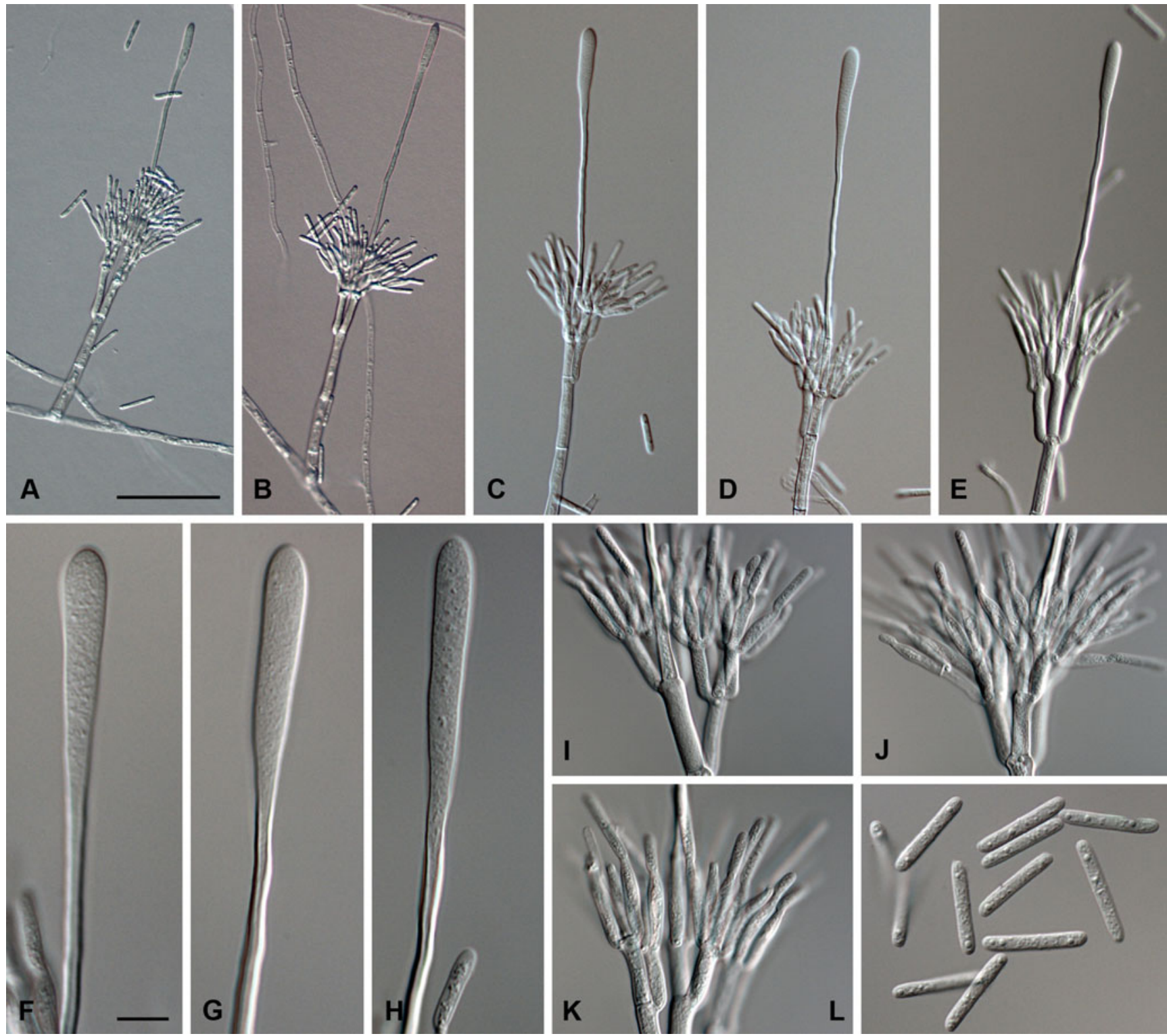

Fig. 15 Cylindrocladiella pseudoparva. a-e. Penicillate conidiophores. $\mathbf{f}-\mathbf{h}$. Terminal vesicles. $\mathbf{i}-\mathbf{k}$. Conidiogenous apparatus with conidiophore branches and phialides. L. Conidia. Scale bars: A=50 $\mu \mathrm{m}$ (apply to $\mathbf{b}-\mathbf{e}$ ), $\mathrm{F}=10 \mu \mathrm{m}$ (apply to $\mathbf{g}-\mathbf{I}$ )

sp., 16 Mar. 1994, L. Petrini, culture CBS 113624=CPC 752.

Notes - Cylindrocladiella pseudoparva can be distinguished from $C$. parva and C. stellenboschensis by having larger primary, and smaller secondary branches. However, phylogenetic inference will be required to accurately identify it. Cylindrocladiella pseudoparva differs from other species in the $C$. parva complex by unique fixed nucleotides in two loci: BTUB position 199 (G) and $358(\mathrm{~A})$; HIS3 position $226(\mathrm{~T})$, 302 (A), 372 (T) and 436 (C).

Cylindrocladiella queenslandica L. Lombard \& Crous, sp. nov. - MycoBank MB561682, Fig. 16.

Etymology - Named after Queensland, the state in Australia from where it was collected.
Cylindrocladiellae infestantis morphologice valde similis, sed conidiis minoribus, (9-)10.5-13.5(-15) $\times 2-4 \mu \mathrm{m}$, distinguitur.

Teleomorph unknown. Conidiophores dimorphic, penicillate and subverticillate, mononematous and hyaline. Penicillate conidiophores (Fig. 16a-e) comprising a stipe, a penicillate arrangement of fertile branches, a stipe extension and a terminal vesicle; stipe septate, hyaline, smooth, 41-82 $\times 6-9 \mu \mathrm{m}$; stipe extension aseptate, straight, 117 $180 \mu \mathrm{m}$ long, thick-walled with one basal septum, terminating in thin-walled, cylindrical to lanceolate vesicles (Fig. 16j-m), 5-8 $\mu \mathrm{m}$ wide. Penicillate conidiogenous apparatus (Fig. 16fi) with primary branches aseptate, $13-23 \times 3-7 \mu \mathrm{m}$, secondary branches aseptate, 9-12×2-4 $\mu \mathrm{m}$, each terminal branch 

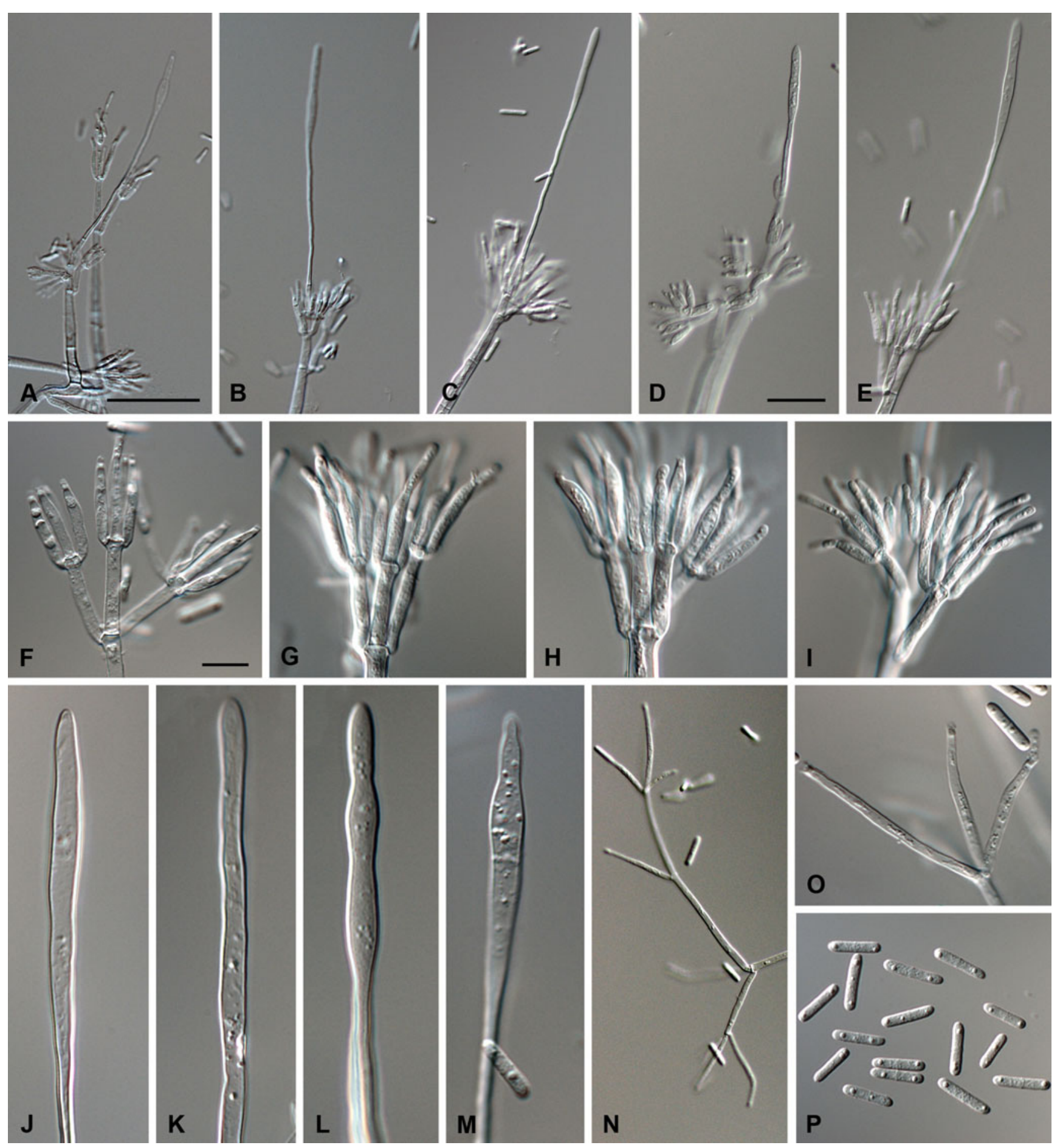

Fig. 16 Cylindrocladiella queenslandica. a-e. Penicillate conidiophores. f-i. Conidiogenous apparatus with conidiophore branches and phialides. $\mathbf{j}-\mathbf{m}$. Terminal vesicles. $\mathbf{n}-\mathbf{o}$. Subverticillate

producing 2-4 phialides; phialides reniform to doliiform to cymbiform, hyaline, aseptate, $7-15 \times 2-4 \mu \mathrm{m}$, apex with minute periclinal thickening and collarette. Subverticillate conidiophores (Fig. 16n-o) in moderate numbers, comprising of a septate stipe, and primary branches terminating in 2-3 conidiophores. P. Conidia. Scale bars: $A=50 \mu \mathrm{m}$ (apply to $\mathbf{b}-\mathbf{c}$ ), $\mathrm{D}=$ $20 \mu \mathrm{m}$ (apply to $\mathbf{e}, \mathbf{n}$ ), $\mathrm{F}=10 \mu \mathrm{m}$ (apply to $\mathbf{g}-\mathbf{m}, \mathbf{0}-\mathbf{p}$ )

phialides; primary branches straight, hyaline, $0-1$-septate, 22 $50 \times 3-4 \mu \mathrm{m}$; phialides cymbiform to cylindrical, hyaline, aseptate, $17-41 \times 2-6 \mu \mathrm{m}$, apex with minute periclinal thickening and collarette. Conidia (Fig. 16p) cylindrical, rounded at both ends, straight, 1-septate, (9-)10.5-13.5 
$(-15) \times 2-4 \mu \mathrm{m}($ av. $=12 \times 3 \mu \mathrm{m})$, frequently slightly flattened at the base, held in asymmetrical clusters by colourless slime.

Culture characteristics - Colonies raised (convex), cottony, with smooth to undulate margins, white with straw (21 d) tint in patches, buff yellow (19 d) to umber (13i) (reverse); chlamydospores moderate throughout medium, arranged in chains; reaching $90 \mathrm{~mm}$ after 7 days on MEA at $24^{\circ} \mathrm{C}$ in the dark.

Specimen examined - Australia, Queensland, from soil, 18 June 2009, P.W. Crous, holotype CBS-H20609, culture ex-type CBS 129574=CPC 17568; Australia, Queensland, from soil, 18 June 2009, P.W. Crous, culture CBS $129575=$ CPC 17569.
Notes - Cylindrocladiella queenslandica can be distinguished from other species in the $C$. infestans complex based on its smaller conidia, and unique fixed nucleotides for three loci: BTUB position 201 (T); HIS3 positions $110(\mathrm{G})$ and $310(\mathrm{G})$; TEF- $1 \alpha$ positions 35 (A) and 455 (T).

Cylindrocladiella stellenboschensis L. Lombard \& Crous, sp. nov. - MycoBank MB561671, Fig. 17.

Etymology - Named after the town from which this species was first collected, Stellenbosch, South Africa.

Cylindrocladiellae parvae morphologice valde similis, sed conidiis majoribus, (14-) 17-19(-21) $\times 2-4 \mu \mathrm{m}$, distinguitur.

Teleomorph unknown. Conidiophores monomorphic, penicillate, mononematous and hyaline. Penicillate
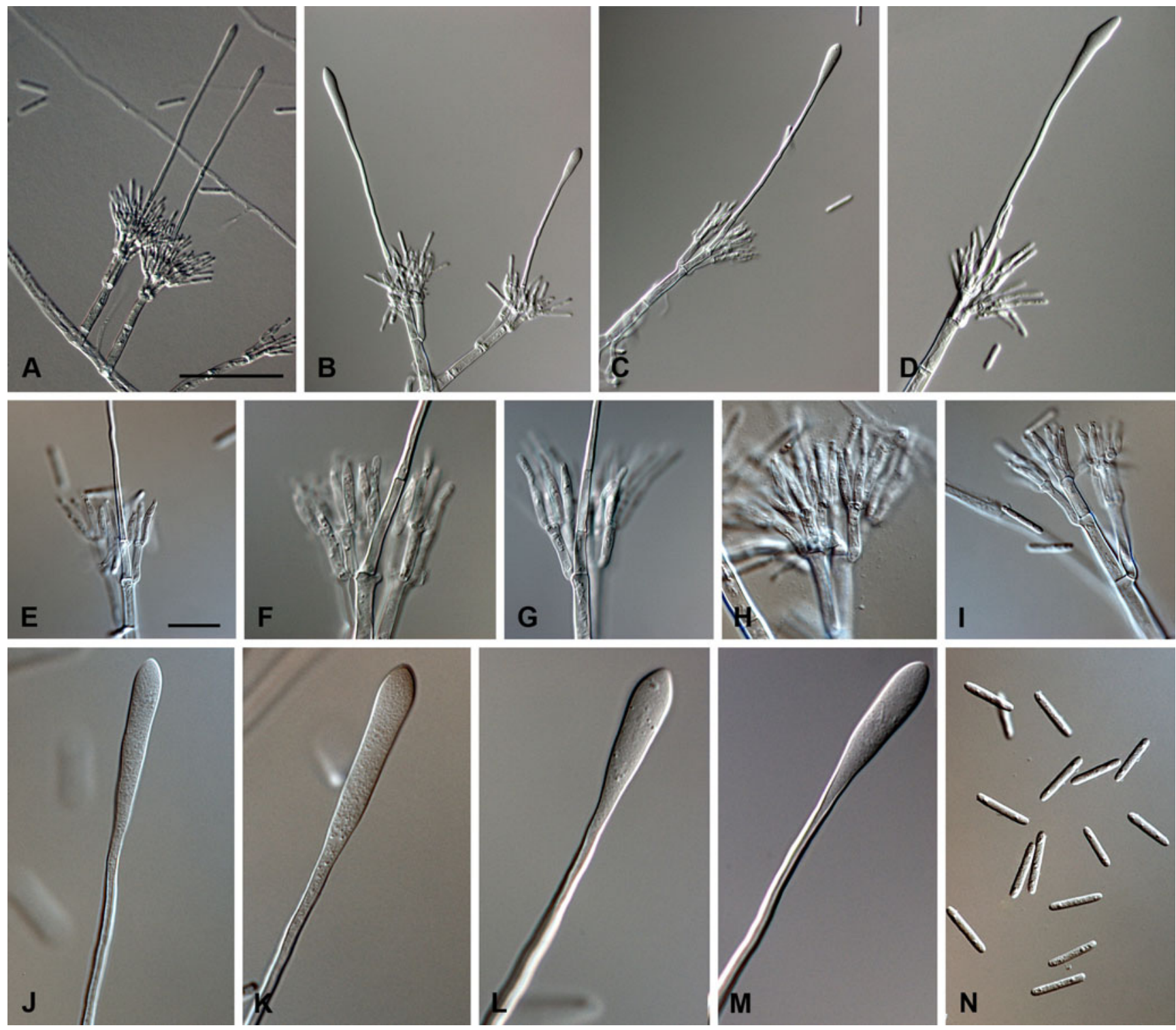

Fig. 17 Cylindrocladiella stellenboschensis a-d. Penicillate conidiophores. e-i. Conidiogenous apparatus with conidiophore branches and phialides. $\mathbf{j}-\mathbf{m}$. Terminal vesicles. N. Conidia. Scale bars: A=50 $\mu \mathrm{m}$ (apply to $\mathbf{b}-\mathbf{d}$ ), $\mathrm{E}=10 \mu \mathrm{m}$ (apply to $\mathbf{f}-\mathbf{n}$ ) 
conidiophores (Fig. 17a-c) comprising a stipe, a penicillate arrangement of fertile branches, a stipe extension and a terminal vesicle; stipe septate, hyaline, smooth, 37$65 \times 6-9 \mu \mathrm{m}$; stipe extension aseptate, straight, 109$169 \mu \mathrm{m}$ long, thick-walled with one basal septum, terminating in thin-walled, clavate to naviculate vesicles (Fig. 17h-j), 5$7 \mu \mathrm{m}$ wide. Penicillate conidiogenous apparatus (Fig. 17d-g) with primary branches aseptate, $13-28 \times 3-5 \mu \mathrm{m}$, secondary branches aseptate, 10-16×3-6 $\mu \mathrm{m}$, each terminal branch producing 2-4 phialides; phialides doliiform to cymbiform, hyaline, aseptate, $12-21 \times 2-4 \mu \mathrm{m}$, apex with minute periclinal thickening and collarette. Subverticillate conidiophores not observed. Conidia (Fig. 17k-n) cylindrical, rounded at both ends, straight, 1-septate, (14-)17-19(-21) $\times$ $2-4 \mu \mathrm{m}(\mathrm{av} .=18 \times 3 \mu \mathrm{m})$, frequently slightly flattened at the base, held in asymmetrical clusters by colourless slime.

Culture characteristics - Colonies raised (convex), cottony, with smooth margins, white with straw (21 d) tint in patches, umber (13i) (reverse); chlamydospores extensive throughout medium, arranged in chains; reaching $60 \mathrm{~mm}$ after 7 days on MEA at $24^{\circ} \mathrm{C}$ in the dark.

Specimens examined - South Africa, Western Cape Province, Stellenbosch, Stellenbosch Botanical Gardens, from leaf litter, 31 Aug. 1992, P.W. Crous, holotype CBSH20610, culture ex-type CBS 110668=CPC 517; Canada, Toronto, Queens Park North, from leaf litter, 24 Apr. 2008, P.W. Crous, culture CPC 15200; Switzerland, Therwil Canton, Basel, from root of Quercus sp., 16 Mar. 1994, L. Petrini, culture CBS 116170=CPC 753.

Notes - This species can be distinguished from C. parva by its larger conidia and shape of the terminal vesicle. Furthermore, collarettes are also present on its phialides, whereas these are rare or absent for C. parva. Cylindrocladiella stellenboschensis differs from other lineages in the $C$. parva complex by unique fixed nucleotides in one locus: BTUB position $112(\mathrm{~A}), 162(\mathrm{G}), 172(\mathrm{~A}), 268(\mathrm{C}), 352(\mathrm{~T}), 361(\mathrm{C}), 366$ (G), $370(\mathrm{~T}), 371(\mathrm{G}), 378(\mathrm{~A}), 382(\mathrm{~A}), 396(\mathrm{~A})$ and $495(\mathrm{C})$.

Cylindrocladiella thailandica L. Lombard \& Crous, sp. nov. - MycoBank MB561680, Fig. 18.

Etymology - Named after the country where it was collected, Thailand.

Cylindrocladiellae infestantis morphologice similis, sed extensionibus stipitis longioribus, $123-183 \mu \mathrm{m}$.

Teleomorph unknown. Conidiophores dimorphic, penicillate and subverticillate, mononematous and hyaline. Penicillate conidiophores (Fig. 18a-d) comprising a stipe, a penicillate arrangement of fertile branches, a stipe extension and a terminal vesicle; stipe septate, hyaline, smooth, 49-80 $\times 5-9 \mu \mathrm{m}$; stipe extension aseptate, straight, $123-$ $183 \mu \mathrm{m}$ long, thick-walled with one basal septum, terminating in thin-walled, cylindrical to lanceolate vesicles (Fig. 18i-m), 5-7 $\mu \mathrm{m}$ wide. Penicillate conidiogenous apparatus (Fig. 18eh) with primary branches aseptate, $11-24 \times 4-8 \mu \mathrm{m}$, secondary branches aseptate, $7-14 \times 2-5 \mu \mathrm{m}$, each terminal branch producing 2-4 phialides; phialides reniform to doliiform to cymbiform, hyaline, aseptate, $8-13 \times 2-4 \mu \mathrm{m}$, apex with minute periclinal thickening and collarette. Subverticillate conidiophores (Fig. 18n-p) in moderate numbers, comprising of a septate stipe, and primary branches terminating in 2-3 phialides; primary branches straight, hyaline, $0-1$-septate, $40 \times 3 \mu \mathrm{m}$; phialides cymbiform to cylindrical, hyaline, aseptate, $19-38 \times 2 \mu \mathrm{m}$, apex with minute periclinal thickening and collarette. Conidia (Fig. 18q) cylindrical, rounded at both ends, straight, 1-septate, $(13-) 14-16(-18) \times 2-4 \mu \mathrm{m}$ (av. = $15 \times 3 \mu \mathrm{m}$ ), frequently slightly flattened at the base, held in asymmetrical clusters by colourless slime.

Culture characteristics - Colonies raised (convex), cottony, with smooth margins, white with buff yellow (19 d) centre, umber (13i) (reverse); chlamydospores extensive throughout medium, arranged in chains; reaching $70 \mathrm{~mm}$ after 7 days on MEA at $24^{\circ} \mathrm{C}$ in the dark.

Specimens examined - Thailand, Chiang Mai, from soil, Oct. 2010, P.W. Crous, holotype CBS-H20611, culture ex-type CBS 129571=CPC 18835; Chiang Mai, from soil, Oct. 2010, P.W. Crous, CBS $129570=$ CPC 18834; Chiang Mai, from soil, Oct. 2010, P.W. Crous, CPC 18831.

Notes - Morphologically, C. thailandica is similar to $C$. infestans and $C$. longistipitata, with the exception that the stipe extensions are longer than those of $C$. infestans but shorter than those of $C$. longistipitata. Cylindrocladiella thailandica can also be distinguished from other species in the $C$. infestans complex by different unique fixed nucleotides for two loci: BTUB position $160(\mathrm{G})$; HIS3 positions 27 (C), 30 (A), 60-63 (indel), 70 (A) and 117 (A).

Cylindrocladiella variabilis L. Lombard \& Crous, sp. nov. - MycoBank MB561673, Fig. 19.

Etymology - Named after its highly variable vesicle morphology.

Cylindrocladiellae lageniformis morphologice similis, sed vesiculis divergentibus.

Teleomorph unknown. Conidiophores monomorphic, penicillate, mononematous and hyaline. Penicillate conidiophores (Fig. 19a-e) comprising a stipe, a penicillate arrangement of fertile branches, a stipe extension and a terminal vesicle; stipe septate, hyaline, smooth, 41-91 $\times 5$ $9 \mu \mathrm{m}$; stipe extension aseptate, straight, 67-106 $\mu \mathrm{m}$ long, thick-walled with one basal septum, terminating in thin-walled, clavate to fusoid to ovoid vesicles (Fig. 19j-m), 5-10 $\mu \mathrm{m}$ wide. Penicillate conidiogenous apparatus (Fig. 19f-i) with primary branches aseptate, 12-23 $\times 3-$ $7 \mu \mathrm{m}$, secondary branches aseptate, $9-14 \times 3-6 \mu \mathrm{m}$, each terminal branch producing $2-4$ phialides; phialides doliiform to cymbiform, hyaline, aseptate, $7-17 \times 2-6 \mu \mathrm{m}$, apex with minute periclinal thickening and collarette absent. Subverticillate conidiophores not observed. Conidia 

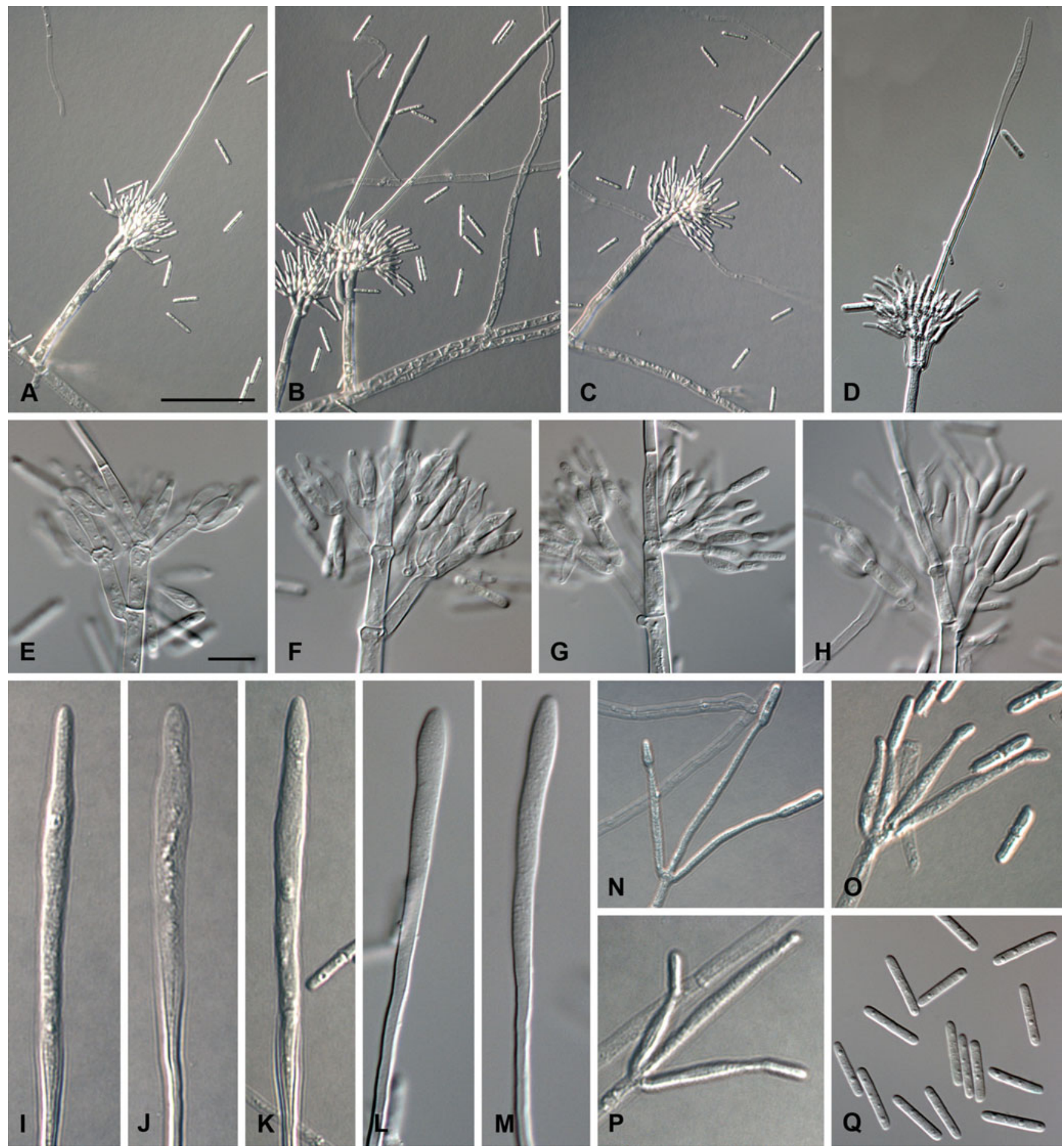

Fig. 18 Cylindrocladiella thailandica a-d. Penicillate conidiophores. $\mathbf{e}-\mathbf{h}$. Conidiogenous apparatus with conidiophore branches and phialides. $\mathbf{i}-$ m. Terminal vesicles. $\mathbf{n}-\mathbf{p}$. Subverticillate conidiophores. Q. Conidia. A=50 $\mu \mathrm{m}$ (apply to $\mathbf{b}-\mathbf{d}$ ), $\mathrm{F}=10 \mu \mathrm{m}$ (apply to $\mathbf{e}-\mathbf{q}$ )

(Fig. 19n) cylindrical, rounded at both ends, straight, 1septate, $(9-) 11-13(-14) \times 2-3 \mu \mathrm{m}(\mathrm{av} .=12 \times 3 \mu \mathrm{m})$, frequently slightly flattened at the base, held in asymmetrical clusters by colourless slime.

Culture characteristics - Colonies raised (convex), cottony, with smooth margins, white, umber (13i) (reverse); chlamydospores extensive throughout medium, arranged in chains; reaching $60 \mathrm{~mm}$ after 7 days on MEA at $24^{\circ} \mathrm{C}$ in the dark.

Specimens examined - Australia, Queensland, Daydream island, Whitsundays Island Resort, from soil, 2 Aug. 2009, P.W. Crous, holotype CBS-H20612, culture ex-type CBS 

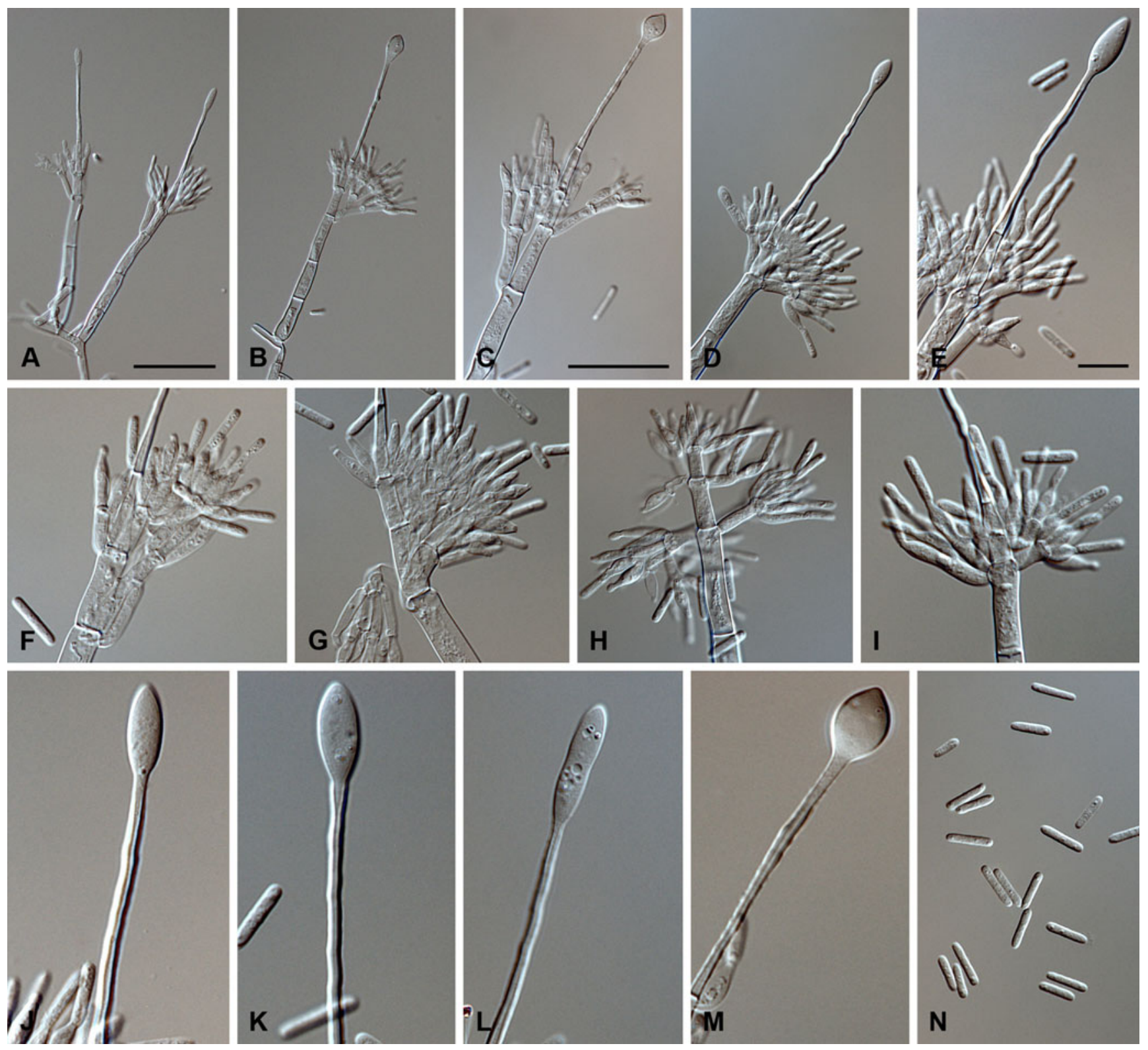

Fig. 19 Cylindrocladiella variabilis. a-e. Penicillate conidiophores. $\mathbf{f}-\mathbf{i}$. Conidiogenous apparatus with conidiophore branches and phialides. $\mathbf{j}-\mathbf{m}$. Terminal vesicles. N. Conidia. Scale bars: $A=20 \mu \mathrm{m}$ (apply to b), $C=50 \mu \mathrm{m}$ (apply to d), $\mathrm{E}=10 \mu \mathrm{m}$ (apply to $\mathbf{f}-\mathbf{n}$ )

$129561=$ CPC 17505, CPC 17504; Australia, Queensland, Lake Barrine, from soil, 18 June 2009, P.W. Crous, culture CBS 129562=CPC 17563.

Notes - Cylindrocladiella variabile can be distinguished from $C$. lageniformis by the high variability of its terminal vesicle shape. This species does not produce subverticillate conidiophores, whereas $C$. lageniformis produces them in moderate numbers (Crous 2002).

Cylindrocladiella pseudoinfestans L. Lombard \& Crous, nom. nov. - MycoBank MB561684

Basionym: Nectricladiella infestans Crous \& C.L. Schoch, Studies in Mycology 45: 55. 2000.
Etymology - Named after its morphological similarity to C. infestans.

Notes - Cylindrocladiella pseudoinfestans is introduced as a new name for $N$. infestans in the genus Cylindrocladiella. Nectricladiella infestans was incorrectly linked to its purported anamorph, C. infestans (Schoch et al. 2000), to which it is morphologically similar. Cylindrocladiella pseudoinfestans can be distinguished from other species in the $C$. infestans complex by different unique fixed nucleotides for three loci: BTUB position 395 (A); HIS3 positions 22 (T), 41 (G), 47 (A), $50(\mathrm{~A}), 72(\mathrm{~T})$ and $272(\mathrm{C})$; TEF-1 $\alpha$ positions $268(\mathrm{~A})$, $272(\mathrm{G}), 478(\mathrm{~A})$ and $480(\mathrm{C})$. 


\section{Discussion}

In this study, several Cylindrocladiella isolates from numerous hosts and countries collected over the past two decades were shown to include a number of novel species. These species were recognised using phylogenetic inference and, where possible, supported by morphological features. The taxonomic status of several phylogenetic species identified in this study remains unresolved due to either representation by only a single isolate (e.g. CBS 116095) or culture sterility (e.g. clade containing CBS 115673). Naming these novel species in the anamorph genus Cylindrocladiella and not the teleomorph genus Nectricladiella follows the "strict priority" option as applied by Gräfenhan et al. (2011), which continued the approach of Lombard et al. (2009, 2010a-c), and Schroers et al. (2011) of naming fungi in the Hyprocreales with the oldest generic name, irrespective of its morph typification. Consequently, the novel species found in this study were named in the genus Cylindrocladiella (Boesewinkel 1982) rather than in the teleomorph genus Nectricladiella (Schoch et al. 2000).

Five species complexes could be identified in this study based on phylogenetic inference supported by morphological characterisation. Although previous authors (Victor et al. 1998, Schoch et al. 2000, Crous 2002, van Coller et al. 2005) acknowledged the presence of species complexes in the genus Cylindrocladiella, their sample sizes were small. In our study, a larger sample size, obtained from various culture collections, allowed a multi-gene analysis to more clearly identify species complexes in Cylindrocladiella.

The Cylindrocladiella camelliae species complex was shown to consist of several phylogenetic species, four of which were described as C. longiphialidica, C. natalensis, C. nederlandica and C. pseudocamelliae. Each of these four new species was distinguished from $C$. camelliae and each other by the morphology and dimensions of conidia, subverticillate conidiophores and stipe extensions. Geographical distribution of the various species in the C. camelliae complex reflected the cosmopolitan nature of this group of fungi. Cylindrocladiella nederlandica and $C$. natalensis were isolated from diseased plant material, and C. pseudocamelliae and $C$. longiphialidica were only isolated from soil, and their significance as plant pathogens still needs to be determined.

Cylindrocladiella cymbiformis is a newly described species closely related to both $C$. novaezelandiae, as well as novel lineages in the newly identified $C$. elegans species complex. Cylindrocladiella cymbiformis is not a cryptic species in the C. elegans complex as it can be distinguished from both $C$. novaezelandiae and $C$. elegans by its larger conidium dimensions and shorter stipe extensions. All isolates in this study representing the $C$. elegans complex originated from South Africa, whereas C. cymbiformis is described here from soil samples collected in Australia. Cryptic species were not resolved in the $C$. elegans complex as the cultures were sterile.

Past studies have presented evidence of cryptic speciation within C. infestans (Victor et al. 1998, Schoch et al. 2000, Crous 2002, van Coller et al. 2005). In an attempt to resolve taxa in this complex, a large sample of $C$. infestans senso lato isolates was included in this study. Based on phylogenetic inference and morphological characterisation, a total of 12 cryptic species were identified. Of these, eight were described as novel taxa. All eight of these newly named species may be regarded as phylogenetic species, as morphological characters are limited to distinguish them from each other. These species are recognised using the genealogical concordance phylogenetic species recognition (GCPSR) criteria (Taylor et al. 2000) based on DNA sequence data for the five loci used in this study. As has been done for other fungal groups (O’Donnell et al. 2004, Grünig et al. 2008, Pavlic et al. 2009, Lombard et al. 2010b), these species are chiefly characterised by fixed single nucleotide polymorphisms (SNPs).

Schoch et al. (2000) described Nectricladiella infestans as the teleomorph state of $C$. infestans sensu lato from an isolate collected in Madagascar that produced perithecia in culture. With additional sequence data and isolates, van Coller et al. (2005) showed this isolate represented a cryptic species distinct from $C$. infestans senso stricto. This was further supported by the phylogenetic inference in this study, and based on GCPSR, Nectricladiella infestans has been provided with a new name, $C$. pseudoinfestans.

Cylindrocladiella clavata, C. lanceolata and C. variabilis are newly described here, closely related to $C$. lageniformis. They can be distinguished from each other and C. lageniformis based on the absence of subverticillate conidiophores, terminal vesicle morphology and conidium dimensions. All three of these species, with the exception of C. lanceolata, are presently only known from soil samples collected in Australia. Cylindrocladiella lanceolata was also isolated from a diseased Eucalyptus cutting in South Africa, adding another Cylindrocladiella species recorded from that country (Crous et al. 1993, Crous et al. 1994, Crous 2002, van Coller et al. 2005).

Phylogenetic inference applied in this study also identified a number of cryptic species within a large sample of $C$. parva sensu lato isolates. Only two of these cryptic species could be named here, as most isolates were sterile. Cylindrocladiella stellenboschensis and C. pseudoparva are difficult to distinguish from each other or from C. parva by morphology alone. These two species are recognised as phylogenetic species described according to the GCPSR criteria using fixed SNPs.

Isolates of $C$. peruviana used in this study also included cryptic species that could not be named. As with the $C$. 
elegans complex, isolates representing these cryptic species were sterile and their taxonomy remains unresolved.

Traditionally, DNA sequence data for the ITS and BTUB gene regions were used to explore the phylogenetic relationship between Cylindrocladiella spp. (Victor et al. 1998, Schoch et al. 2000). Van Coller et al. (2005) introduced HIS3 sequence data for this group of fungi, increasing the gene regions that provide the most valuable information on the relationships among Cylindrocladiella spp. Data for these three gene regions have been available only for a small sample of Cylindrocladiella isolates. This present study has attempted to address this problem and also introduced partial TEF- $1 \alpha$ gene region sequences for all known Cylindrocladiella spp. Phylogenetic analysis of the individual gene regions showed that the TEF- $1 \alpha$ gene region provided the best resolution to distinguish between Cylindrocladiella spp., followed by BTUB and HIS3. As was found with Calonectria spp. (Lombard et al. 2010b), the ITS and LSU gene regions provided limited information to distinguish between Cylindrocladiella spp.

Identification of a large number of cryptic species within the genus Cylindrocladiella based on phylogenetic inference and morphological comparisons, highlights how little attention this group has received in the past. Although Cylindrocladiella spp. are generally not regarded as important plant pathogens, correct identification is essential for disease control and biosecurity implications. This study has revealed the importance of combining morphological and phylogenetic data to understand the taxonomic issues surrounding this group of fungi.

Acknowledgments Prof. dr U. Braun (Martin-Luther-Univ., Halle, Germany) is thanked for providing the Latin diagnoses. The authors thank the technical staff, A. van Iperen (cultures), M. Vermaas (photographic plates), for their invaluable assistance.

Open Access This article is distributed under the terms of the Creative Commons Attribution Noncommercial License which permits any noncommercial use, distribution, and reproduction in any medium, provided the original author(s) and source are credited.

\section{References}

Boesewinkel HJ (1982) Cylindrocladiella, a new genus to accommodate Cylindrocladium parvum and other small-spored species of Cylindrocladium. Can J Bot 60:2288-2294

Crous PW (2002) Taxonomy and pathology of Cylindrocladium (Calonectria) and allied genera. APS Press, St. Paul, Minnesota, USA

Crous PW, Wingfield MJ (1993) A re-evaluation of Cylindrocladiella, and a comparison with morphologically similar genera. Mycol Res 97:433-448

Crous PW, Phillips AJL, Wingfield MJ (1991) The genera Cylindrocladium and Cylindrocladiella in South Africa, with special reference to forest nurseries. S Afr J For 157:69-85
Crous PW, Wingfield MJ, Lennox CL (1994) A comparison of generic concepts in Calonectria and Nectria with anamorphs in Cylindrocladium and Cylindrocladiella. S Afr J Sci 90:485-488

Crous PW, Gams W, Stalpers JA, Robert V, Stegehuis G (2004) MycoBank: an online initiative to launch mycology into the $21^{\text {st }}$ century. Stud Mycol 50:19-22

Crous PW, Slipper B, Wingfield MJ, Rheeder J, Marasas WFO, Phillips AJL, Alves A, Burgess T, Barber P, Groenewald JZ (2006) Phylogenetic lineages in the Botryosphaeriaceae. Stud Mycol 55:235-253

Crous PW, Wood AR, Okada G, Groenewald JZ (2008) Foliicolous microfungi occurring on Encephalartos. Persoonia 21:135-146

Crous PW, Summerell BA, Carnegie AJ, Wingfield MJ, Groenewald JZ (2009) Novel species of Mycosphaerellaceae and Teratosphaeriaceae. Persoonia 23:119-146

Gräfenhan T, Schroers H-J, Nirenberg HI, Seifert KA (2011) An overview of the taxonomy, phylogeny, and typification of nectriaceous fungi in Cosmospora, Acremonium, Fusarium, Stilbella and Volutella. Stud Mycol 68:79-114

Grünig CR, Duò A, Sieber TN, Holdenrieder O (2008) Assignment of species rank to six reproductively isolated cryptic species of the Phialocephala fortinii s.1.-Acephala applanata species complex. Mycologia 100:47-67

Gueidan C, Roux C, Lutzoni F (2007) Using multigene phylogeny analysis to assess generic delineation and character evolution in Verrucariaceae (Verrucariales, Ascomycota). Mycol Res 111:11451168

Katoh K, Toh H (2008) Recent developments in the MAFFT multiple sequence alignment program. Brief Bioinform 9:286-298

Lombard L, Rodas CA, Crous PW, Wingfield BD, Wingfield MJ (2009) Cylindrocladium species associated with dying Pinus cuttings. Persoonia 23:41-47

Lombard L, Crous PW, Wingfield BD, Wingfield MJ (2010a) Multigene phylogeny and mating tests reveal three cryptic species related to Calonectria pauciramosa. Stud Mycol 66:15-30

Lombard L, Crous PW, Wingfield BD, Wingfield MJ (2010b) Phylogeny and systematics of the genus Calonectria. Stud Mycol 66:31-69

Lombard L, Zhou XD, Crous PW, Wingfield BD, Wingfield MJ (2010c) Calonectria species associated with cutting rot of Eucalyptus. Persoonia 24:1-11

Möller EM, Bahnweg G, Sandermann H, Geiger HH (1992) A simple and efficient protocol for isolation of high molecular weight DNA from filamentous fungi, fruitbodies, and infected plant tissue. Nucleic Acids Res 20:6115-6116

Nirenburg HI (1981) A simplified method to identify Fusarium spp. occurring on wheat. Can J Bot 59:1599-1609

Nylander JAA (2004) MrModeltest v. 2. Programme distributed by the author. Evolutionary Biology Centre, Uppsala University

O'Donnell K, Ward TJ, Geiser DM, Kistler HC, Aoki T (2004) Genealogical concordance between the mating type locus and seven other nuclear genes supports formal recognition of nine phylogenetically distinct species within the Fusarium graminearum clade. Fungal Genet Biol 41:600-623

Pavlic D, Slippers B, Coutinho TA, Wingfield MJ (2009) Molecular and phenotypic characterization of three phylogenetic species discovered within the Neofusicoccum parvum/N. ribis complex. Mycologia 101:636-647

Peerally A (1991) The classification and phytopathology of Cylindrocladium species. Mycotaxon 40:323-366

Rayner RW (1970) A mycological colour chart. Commonwealth Mycological Society, Kew, Surrey. British Mycological Society

Ronquist F, Heulsenbeck JP (2003) MrBayes 3: Bayesian phylogenetic inference under mixed models. Bioinformatics 19:1572-1574 
Scattolin L, Montecchio L (2007) First report of damping-off of common oak plantlets caused by Cylindrocladiella parva in Italy. Plant Dis 91:771

Schoch CL, Crous PW, Wingfield MJ, Wingfield BD (2000) Phylogeny of Calonectria and selected hypocrealean genera with cylindrical macroconidia. Stud Mycol 45:45-62

Schroers H-J, Gräfenhan T, Nirenberg HI, Seifert KA (2011) A revision of Cyanonectria and Geejayessia gen. nov., and related species with Fusarium-like anamorphs. Stud Mycol 68:115-138

Sharma JK, Mohanan C (1991) In vitro evaluation of fungicides against Cylindrocladium spp. causing diseases of Eucalyptus in Kerala, India. Eur J Forest Pathol 21:17-26
Swofford DL (2002) PAUP*. Phylogenetic analysis using parsimony (* and other methods), v. 4.0b10. Computer program. Sunderland, Massachusetts, USA: Sinauer Associates

Taylor JW, Jacobson DJ, Kroken S, Kasuga T, Geiser DM, Hibbett DS, Fisher MC (2000) Phylogenetic species recognition and species concepts in fungi. Fungal Genet Biol 31:21-32

Van Coller GJ, Denman S, Groenewald JZ, Lamprecht SC, Crous PW (2005) Characterisation and pathogenicity of Cylindrocladiella spp. associated with root and cutting rot symptoms of grapevines in nurseries. Australas Plant Path 34:489-498

Victor D, Crous PW, Janse BJH, van Zyl WH, Wingfield MJ, Alfenas AC (1998) Systematic appraisal of species complexes within Cylindrocladiella. Mycol Res 102:273-279 